Beitrag / Article

Open Access

Susanne Krings*

\title{
Doppelt relevant: Kritische Infrastrukturen der Daseinsvorsorge
}

\section{Doubly relevant: Critical infrastructures of Daseinsvorsorge}

https://doi.org/10.2478/rara-2020-0034

Eingegangen: 19. April 2020, Angenommen: 17. November 2020

Kurzfassung: Die räumliche Planung ist aufgerufen, sich im Rahmen des Schutzes kritischer Infrastrukturen am Umgang mit dem Risiko von Ausfällen als besonders wichtig geltender Infrastrukturleistungen zu beteiligen. In diesem Beitrag werden Hinweise auf Überschneidungen zwischen den Sektoren kritischer Infrastrukturen und Bereichen der Daseinsvorsorge untersucht, denen in der fachöffentlichen Diskussion bislang wenig Aufmerksamkeit zuteil wurde. Den Sektoren kritischer Infrastrukturen werden dazu unterschiedliche Auffassungen von Bereichen der Daseinsvorsorge gegenübergestellt. Auch wenn sich die Überschneidungsbereiche nicht genau bestimmen lassen, ist davon auszugehen, dass ein Großteil der als kritisch betrachteten Infrastrukturen an der Bereitstellung von Dienstleistungen beteiligt ist, die der Daseinsvorsorge zugerechnet werden. Die betreffenden Infrastrukturen und Dienstleistungen sind demnach Gegenstand unterschiedlicher politischer Gestaltungsbereiche und darauf bezogener Maßnahmen. Diese Folgerung lädt zur Befassung mit den Implikationen der räumlichen Organisation der Bereitstellung von Daseinsvorsorgeleistungen für den Schutz kritischer Infrastrukturen ein. Ein Perspektivwechsel, die Betrachtung von Zielen und Ansätzen der Daseinsvorsorgeplanung aus der Sicht des Schutzes kritischer Infrastrukturen, kann deren mögliche ,Nebenwirkungen“ sichtbar machen. Da das Konzept „kritische Infrastruktur“ Schutzwürdigkeit und Gefahrenpotenzial auf sich vereinigt, eröffnet ein solcher Perspektivwechsel mitunter ungewohnte Ansichten auf die Daseinsvorsorgeplanung und wirft Fragen für Forschung und Praxis auf.

Schlüsselwörter: Daseinsvorsorge, Deutschland, kritische Infrastruktur, räumliche Planung, Risikomanagement

Abstract: Spatial planning is called on to contribute to managing risks associated with outages of infrastructure services deemed particularly important in the context of critical infrastructure protection. This paper explores indications of overlaps between critical infrastructure sectors and the fields of Daseinsvorsorge (services of general interests) which have not yet received much attention in expert discussions. It provides a comparison of critical infrastructure sectors and variants of lists of the fields of Daseinsvorsorge. Although the extent of the overlaps cannot be determined exactly, it can be assumed that most of the infrastructures considered to be critical are involved in the provision of services associated with the concept of Daseinsvorsorge. Hence the infrastructures and services in question are subject to different policy areas and interventions carried out in accordance with them. This inference calls for consideration of the implications of the spatial organization of the provision of Daseinsvorsorge services for critical infrastructure protection. A change of perspective, i.e. looking at the aims and means of Daseinsvorsorge planning from the point of view of critical infrastructure protection, enhances the visibility of their potential 'side effects'. As the

\footnotetext{
*Corresponding author: Susanne Krings, Rheinische Friedrich-Wilhelms-Universität Bonn, Geographisches Institut, Geographische Entwicklungsforschung, Meckenheimer Allee 166, 53115 Bonn, Deutschland, E-Mail: susanne.krings@uni-bonn.de

Die Autorin ist Mitarbeiterin des Bundesamts für Bevölkerungsschutz und Katastrophenhilfe, vertritt hier aber ausschließlich ihre persönliche Auffassung.
} 
concept of critical infrastructure incorporates both worthiness of protection and a certain hazard potential, a change of perspective leads to unfamiliar perceptions of the spatial planning of Daseinsvorsorge and raises academic and practical questions.

Keywords: Services of general interest, Germany, Critical infrastructure, Spatial planning, Risk management

\section{Einleitung}

Im Rahmen des Schutzes kritischer Infrastrukturen werden Risiken im Zusammenhang mit „gravierende[n] Störungen und Ausfälle[n] von wichtigen Infrastrukturleistungen" (BMI 2009: 10) adressiert. ${ }^{1}$ Entsprechende Ereignisse sollen einer Risikomanagement-Logik folgend möglichst vermieden und deren Folgen im Bedarfsfall minimiert werden (vgl. BMI 2009: 10). Spätestens seit im Jahr 2008 der Passus „dem Schutz kritischer Infrastrukturen ist Rechnung zu tragen" (§ 2 Abs. 2 Nr. 3 ROG) $)^{2}$ in die Grundsätze der Raumordnung aufgenommen wurde, stellt sich die Frage, wie die räumliche Planung zum Risikomanagement beitragen kann. In der Auseinandersetzung mit dieser Frage wurde eine Aussage aus dem Raumordnungsbericht 2011 bislang wenig beachtet: „Die meisten Bereiche der öffentlichen Daseinsvorsorge", so heißt es dort (BBSR 2012: 53), „lassen sich einem Sektor oder Teilsektor der Kritischen Infrastrukturen zuordnen." Der vorliegende Beitrag geht diesem Hinweis (und ähnlichen, vgl. BBK 2019b: 17) nach und bietet damit der fachöffentlichen Diskussion um die Berücksichtigung kritischer Infrastrukturen in der räumlichen Planung einen weiteren Ansatzpunkt an. Lassen sich also „die meisten Bereiche der öffentlichen Daseinsvorsorge [...] einem Sektor oder Teilsektor der Kritischen Infrastrukturen zuordnen“ (BBSR 2012:

1 Der Begriff "Risiko” ist vielfach definiert worden und es haben sich spezifische Varianten eines Risikomanagement-Fachvokabulars herausgebildet. Die Verwendung der Begriffe im vorliegenden Beitrag orientiert sich an der im behördlichen Umfeld üblichen Terminologie (vgl. BBK 2019a). Risiken erwachsen danach aus der Möglichkeit, dass eine abstrakte "Gefahr" die Form eines konkreten „Ereignisses“ annimmt, wodurch Schäden an „Schutzgütern“ entstehen können. „Risiko“ wird verstanden als „Kombination aus der Eintrittswahrscheinlichkeit eines Ereignisses und dessen negativen Folgen“ (BBK 2019a: 45). „Risikomanagement“ bezeichnet den systematischen Umgang mit Risiken von der Analyse und Bewertung bis zur Umsetzung von Maßnahmen. Maßnahmen zur Risikominderung können an der Absenkung der Eintrittswahrscheinlichkeit eines Ereignisses oder der Reduzierung des zu erwartenden Schadensausmaßes ansetzen, z. B. indem sie die Exposition oder die Verwundbarkeit von Schutzgütern herabsetzen.

2 Raumordnungsgesetz (ROG) vom 22. Dezember 2008, zuletzt geändert durch Artikel 2 Absatz 15 des Gesetzes vom 20. Juli 2017.
53)? Werden womöglich dieselben Infrastrukturen, die als kritische Infrastrukturen gelten, auch als Infrastrukturen der Daseinsvorsorge betrachtet und als solche zum Gegenstand (raumordnungs)politischer Entscheidungen und (raum)planerischer Maßnahmen? Und, sollte dem so sein, wie kann davon ausgehend über den Umgang mit kritischen Infrastrukturen in der räumlichen Planung nachgedacht werden?

Um sich diesen Fragen zu nähern, stellt Kapitel 2 zunächst auf der Basis zentraler Politikinstrumente Kernaspekte des Schutzes kritischer Infrastrukturen in Deutschland vor, gefolgt von einem Überblick über die in behördlichen und fachwissenschaftlichen Veröffentlichungen bereits thematisierter Ansätze, die Interessen des Schutzes kritischer Infrastrukturen in der räumlichen Planung zu berücksichtigen (Kapitel 3). Kapitel 4 führt Daseinsvorsorge als politisches Konzept ein, bevor Kapitel 5 die Aussage zu Überschneidungen zwischen Bereichen der Daseinsvorsorge und Sektoren oder Teilsektoren kritischer Infrastrukturen zu überprüfen versucht. Dazu werden den Sektoren kritischer Infrastrukturen unterschiedliche Auffassungen von den Bereichen der Daseinsvorsorge gegenübergestellt und die so gewonnenen Befunde diskutiert. Kapitel 6 lädt auf dieser Grundlage zu einem Perspektivwechsel ein: Es wird die Frage aufgeworfen, wie sich die Sicht auf die vorgefundene oder angestrebte Struktur der räumlichen Organisation von Versorgung ändert, wenn deren möglicher Ausfall zum Ausgangspunkt gemacht wird. Anhand von Beispielen wird der Blick auf mögliche Implikationen der räumlichen Daseinsvorsorgeplanung für den Schutz kritischer Infrastrukturen gelenkt und Ansätze zu deren weiterer Untersuchung aufgezeigt. Kapitel 7 schließt mit einem Resümee $a b$.

\section{Schutz kritischer Infrastrukturen: vom Risiko des Versorgungsausfalls}

Seit seinen Anfängen Ende der 1990er-Jahre hat sich der Schutz kritischer Infrastrukturen in Deutschland aus- 
gehend von der Bundesebene als Politikfeld etabliert (vgl. Schulze 2006: 155-159, 205-219; BBK 2020: 17-19). Die ressortübergreifende Koordinierung dieser „Kernaufgabe staatlicher Sicherheitsvorsorge" (BMI 2009: 3) liegt beim Bundesinnenministerium (BMI), unterstützt durch Behörden in seinem Geschäftsbereich, insbesondere das Bundesamt für Bevölkerungsschutz und Katastrophenhilfe (BBK) und das Bundesamt für Sicherheit in der Informationstechnik (BSI). Politisch-strategische Arbeitsgrundlage ist die 2009 vom Bundeskabinett beschlossene „Nationale Strategie zum Schutz Kritischer Infrastrukturen" (BMI 2009). Sie wird von Instrumenten flankiert, die sich einem ebenen- und akteursübergreifenden Aufgabenverständnis folgend an unterschiedliche Zielgruppen richten, vor allem an Infrastrukturbetreiber und Behörden in Bund, Ländern und Kommunen (z. B. BMI 2005; BMI 2011; BBK 2012; BBK 2019b). ${ }^{3}$

Als „kritische Infrastrukturen"4 werden "Organisationen und Einrichtungen mit wichtiger Bedeutung für das staatliche Gemeinwesen“ bezeichnet, „bei deren Ausfall oder Beeinträchtigung nachhaltig wirkende Versorgungsengpässe, erhebliche Störungen der öffentlichen Sicherheit oder andere dramatische Folgen eintreten würden“ (BMI 2009: 3). Als spezifisches Merkmal kritischer Infrastrukturen gilt ihre hohe „Kritikalität" verstanden als „relatives Maß für die Bedeutsamkeit einer Infrastruktur in Bezug auf die Konsequenzen, die eine Störung oder ein Funktionsausfall für die Versorgungssicherheit der Gesellschaft mit wichtigen Gütern und Dienstleistungen hat" (BMI 2009: 5). Die Verknüpfung von Bedeutsamkeit und Konsequenzen bei Ausfall macht Kritikalität zu einem vielschichtigen Konzept: Einerseits kommt darin Schutzwürdigkeit zum Ausdruck, andererseits Gefahrenpotenzial. Zudem wird Kritikalität als Merkmal einer Infrastruktur ${ }^{6}$ verstanden, sie manifes-

3 Vgl. auch BSI-KritisV - BSI-Kritisverordnung vom 22. April 2016, zuletzt geändert durch Artikel 1 der Verordnung vom 21. Juni 2017, und IT-SiG - IT-Sicherheitsgesetz vom 17. Juli 2015.

4 Der Begriffsbestandteil „kritisch“ wird in Quellen und der Fachliteratur uneinheitlich groß- oder kleingeschrieben. Im Folgenden wird die Kleinschreibung bevorzugt und in Zitaten die im Original verwendete Schreibweise beibehalten.

5 Zum Begriff „Kritikalität“ vgl. Engels (2018), Folkers (2018b) und Lukitsch/Müller/Stahlhut (2018).

6 Der Begriff "Infrastruktur" bleibt in den Quellen zuweilen unscharf. Da der Begriff „kritische Infrastrukturen“ im Plural definiert wird, ist unklar, ob er sich im Singular auf ein Infrastrukturelement (z. B. ein Umspannwerk) oder einen Infrastrukturbereich (z. B. die Gesamtheit der Infrastrukturelemente zur Stromversorgung) bezieht. Die ausgewerteten Quellen lassen beide Interpretationen zu. Auch „Kritikalität“ wird mitunter auf ganze Infrastrukturbereiche und weitere Kategorien wie Dienstleistungen oder einzelne Prozesse tiert sich allerdings in den Folgen ihres Ausfalls für die "Versorgungssicherheit der Gesellschaft“ (BMI 2009: 5) und verweist damit auf ein über die Infrastruktur hinausweisendes Beziehungsgefüge. Die kritische Infrastruktur ist demnach Schutzobjekt und Gefahrenquelle, wobei sich beides aus ihrer Position in einem größeren Versorgungszusammenhang ergibt.

Für jene „wichtigen Infrastrukturleistungen“, deren Störung oder Ausfall problematisiert werden, hat sich in den letzten Jahren der Begriff „kritische Dienstleistung“ durchgesetzt. Er bezeichnet eine „Dienstleistung, die von Betreibern Kritischer Infrastrukturen zur Versorgung der Allgemeinheit erbracht wird und deren Ausfall oder Beeinträchtigung zu erheblichen Versorgungsengpässen, zu Gefährdungen der öffentlichen Sicherheit oder zu vergleichbaren Folgen führen würde" (BBK 2019a: 34) oder schlicht eine Dienstleistung, die „essentiell für die Versorgung der Bevölkerung" ist (BBK 2019b: 36). Die Verwendung des Begriffs dokumentiere, so BBK (2020: 25), „eine gewisse Akzentverschiebung - vom Schutz der Infrastruktur zur Aufrechterhaltung der Dienstleistung". Kritischen Infrastrukturen wird eine zentrale Rolle bei der Bereitstellung kritischer Dienstleistungen beigemessen und der Schutz der Infrastruktur als Mittel zur Aufrechterhaltung der Dienstleistung aufgefasst. Eine Übersicht zum "derzeitigen Diskussionsstand“ (BBK 2020: 25) hinsichtlich dessen, welche Dienstleistungen zu den kritischen Dienstleistungen zu zählen sind, ist Tabelle 1 zu entnehmen.

Bevor das Konzept der kritischen Dienstleistungen seine inzwischen sehr zentrale Stellung innerhalb der Begriffssystematik einnahm, wurde der Gegenstandsbereich des Schutzes kritischer Infrastrukturen bereits über die Nennung von Infrastrukturbereichen, den „Sektoren", konkretisiert. Die mehrfache Überarbeitung der Sektorenliste in den letzten zwei Jahrzehnten zeugt von fortlaufenden Diskussionen um die Ausrichtung des Politikfeldes (BBK 2020: 22-23; vgl. Krings 2018: 107-108). In der aktuellen, seit 2011 zwischen Bund und Ländern abgestimmten Fassung werden insgesamt neun Sektoren unterschieden, die auf der Bundesebene zusätzlich in 29 Branchen untergliedert sind. Inzwischen werden die kritischen Dienstleistungen, wie in Tabelle 1 dargestellt, den Sektoren zugeordnet (vgl. BBK 2019b: 38; BBK 2020: 24).

Die Unterteilung in Sektoren und Branchen impliziert nicht, dass es sich um separate Systeme handelt. Vielmehr wird beim Schutz kritischer Infrastrukturen von „hohen Interdependenzen zwischen einzelnen Inf-

angewendet. 
Tabelle 1: Sektoren und Branchen kritischer Infrastrukturen sowie kritische Dienstleistungen (BBK 2020: 24)

\begin{tabular}{|c|c|c|}
\hline $\begin{array}{l}\text { Sektoren } \\
\text { (Bund und Länder) }\end{array}$ & $\begin{array}{l}\text { Branchen } \\
\text { (Bund) }\end{array}$ & $\begin{array}{l}\text { Kritische Dienstleistungen } \\
\text { (Diskussionsstand) }\end{array}$ \\
\hline Energie & Elektrizität, Mineralöl, Gas & $\begin{array}{l}\text { Stromversorgung, Gasversorgung, Kraftstoff- und } \\
\text { Heizölversorgung, Fernwärmeversorgung }\end{array}$ \\
\hline Ernährung & Ernährungswirtschaft, Lebensmittelhandel & Lebensmittelversorgung \\
\hline $\begin{array}{l}\text { Finanz- und } \\
\text { Versicherungswesen }\end{array}$ & $\begin{array}{l}\text { Banken, Börsen, Versicherungen, } \\
\text { Finanzdienstleister }\end{array}$ & $\begin{array}{l}\text { Zahlungsverkehr, Bargeldversorgung, } \\
\text { Kreditvergabe, Wertpapier- und Derivatehandel, } \\
\text { Versicherungsdienstleistungen }\end{array}$ \\
\hline Gesundheit & $\begin{array}{l}\text { Medizinische Versorgung, Arzneimittel und } \\
\text { Impfstoffe, Labore }\end{array}$ & $\begin{array}{l}\text { Medizinische Versorgung, Versorgung mit } \\
\text { Arzneimitteln (einschließlich Impfstoffen und } \\
\text { Schutzwirkstoffen nach Strahlenschutzrecht), } \\
\text { Versorgung mit Medizinprodukten, } \\
\text { Laboratoriumsdiagnostik }\end{array}$ \\
\hline $\begin{array}{l}\text { Informationstechnik und } \\
\text { Telekommunikation }\end{array}$ & Telekommunikation, Informationstechnik & $\begin{array}{l}\text { Leitungsgebundene und ungebundene (auch } \\
\text { weltraumbasierte) Sprach- und Datenübertragung, } \\
\text { Datenspeicherung und -verarbeitung }\end{array}$ \\
\hline Medien und Kultur & $\begin{array}{l}\text { Rundfunk (Fernsehen und Radio), gedruckte } \\
\text { und elektronische Presse, Kulturgut, } \\
\text { symbolträchtige Bauwerke }\end{array}$ & $\begin{array}{l}\text { Warnung und Alarmierung, Versorgung mit } \\
\text { Informationen, Herstellen von Öffentlichkeit, } \\
\text { Aufbewahrung identitätsstiftender } \\
\text { Kulturgegenstände und Dokumente, Vermittlung } \\
\text { kultureller Identität, Langzeitsicherung und } \\
\text {-lagerung von mikroverfilmten Dokumenten der } \\
\text { deutschen Geschichte gemäß Haager Konvention } \\
\text { zum Schutz von Kulturgut }\end{array}$ \\
\hline Staat und Verwaltung & $\begin{array}{l}\text { Regierung und Verwaltung, Parlament, } \\
\text { Justizeinrichtungen, Notfall- und Rettungswesen } \\
\text { einschließlich Katastrophenschutz }\end{array}$ & $\begin{array}{l}\text { Umsetzung von Recht im Rahmen der Eingriffs- } \\
\text { und Leistungsverwaltung, (polizeiliche und } \\
\text { nichtpolizeiliche) Gefahrenabwehr, Verteidigung, } \\
\text { Gesetzgebung, Kontrolle der Regierung, } \\
\text { Rechtsprechung und deren Vollzug }\end{array}$ \\
\hline Transport und Verkehr & $\begin{array}{l}\text { Luftfahrt, Seeschifffahrt, Binnenschifffahrt, } \\
\text { Schienenverkehr, Straßenverkehr, Logistik }\end{array}$ & $\begin{array}{l}\text { Leistungen zum Transport von Personen, } \\
\text { Leistungen zum Transport von Gütern, } \\
\text { Satellitennavigationssysteme und } \\
\text { satellitengestützte Positions-, Navigations- und } \\
\text { Zeit- sowie meteorologische Dienste }\end{array}$ \\
\hline Wasser & $\begin{array}{l}\text { Öffentliche Wasserversorgung und öffentliche } \\
\text { Abwasserbeseitigung }\end{array}$ & Trinkwasserversorgung, Abwasserbeseitigung \\
\hline
\end{tabular}

rastruktursystemen“ (BMI 2009: 7) ausgegangen, durch die sich die Auswirkungen von Ausfällen in Form von "Domino- und Kaskadeneffekten“ ausbreiten können. Der Begriff „Interdependenz" wird uneinheitlich verwendet - meist für Abhängigkeiten über die Branchen- und Sektorengrenzen hinweg, zuweilen auch als Sammelbegriff für diverse Formen von Abhängigkeitsbeziehungen auf unterschiedlichen Ebenen (z. B. BMI 2005: 51; BMI 2009: 5, 7; BMI 2011: 10; BBK 2012: 25). ${ }^{7}$ Interdepen-

$7 \mathrm{Zu}$ unterschiedlichen Formen von Interdependenz und ihrer Operationalisierung vgl. Rinaldi/Peerenboom/Kelly (2001), Hempel/ Kraff/Pelzer (2018), inter3 (2019) und Schmitt (2019). denzen stellen eine Art „endogenes Störungspotenzial“ (Folkers 2018a: 162) in den vernetzten Infrastruktursystemen dar. Sie wirken als Ausbreitungspfade für die Folgen von Störungen (vgl. Folkers 2012: 157-158) und gelten dementsprechend als zentraler Verwundbarkeitsfaktor (BMI 2009: 7; vgl. Folkers 2018a: 161-164).

Die Konstellation aus kritischen Infrastrukturen und kritischen Dienstleistungen spiegelt sich in der Konstruktion von Gefahren und Schutzgütern auf unterschiedlichen Ebenen wider. Dem in der Strategie beschriebenen „All-Gefahren-Ansatz" (BMI 2009: 7) nach reicht das Gefahrenspektrum für kritische Infrastrukturen von Naturereignissen bis zu kriegerischen Auseinanderset- 
zungen. Der Schutz der kritischen Infrastrukturen vor „allen“ Gefahren dient der unterbrechungsfreien Bereitstellung der kritischen Dienstleistungen, deren Ausfall wiederum als Gefahr für die Bevölkerung sowie weiterer abstrakter Werte, wie „unsere[r] Lebensweise“ (BBK 2012: 13) oder der "öffentlichen Sicherheit“ (BMI 2009: $3)$, gilt. Die Parallelität von Auslöser und Ausfall auf der Gefahrenseite korrespondiert mit den jeweils adressierten Schutzgütern, sodass sich der Schutz kritischer Infrastrukturen als Risikomanagementprozess auf mehreren, miteinander verbundenen Ebenen darstellt.

Neben der Verwundbarkeit der betreffenden Infrastrukturen wird dementsprechend auch eine hohe gesellschaftliche Verwundbarkeit gegenüber Ausfällen kritischer Dienstleistungen als Problem bewertet: Gewohnt an die zuverlässige Versorgung habe sich ein "durchaus trügerisches Gefühl von Sicherheit" eingestellt, weswegen "die Auswirkungen eines ,Dennoch-Störfalls‘ überproportional hoch“ (BMI 2009: 8) ausfallen würden. Der Zusammenhang zwischen immer selteneren Ausfällen und immer schwerwiegenderen Folgen wird als „Verletzlichkeitsparadoxon" (BMI 2009: 8) bezeichnet. Das Risikomanagement steckt dadurch in einem Dilemma: Die Zuverlässigkeit der Versorgung, die es einerseits weiter zu erhöhen gilt, ist andererseits auch Teil des Problems (vgl. Krings 2018: 110-111).

Hinsichtlich der Umsetzung von Maßnahmen zum Schutz der kritischen Infrastrukturen werden deren Betreiber in einer zentralen Rolle gesehen (vgl. BMI 2005: 6; BBK 2012: 24; BBK 2020: 22). Da es sich dabei mehrheitlich und in weiter steigendem Maße um private bzw. privatrechtliche Akteure handele, gehe die „Verantwortung für die Sicherheit, Zuverlässigkeit und Verfügbarkeit dieser Infrastrukturen zunehmend in private, zumindest aber geteilte Verantwortung über" (BMI 2009: 6). Aufgaben der öffentlichen Hand bewegten sich unter diesen Bedingungen „vorrangig im Rahmen einer Gewährleistung, allenfalls in der Sicherstellung der Versorgung in Krisenzeiten" (BMI 2009: 6). ${ }^{8}$ Die Wettbewerbssituation, in der sich Infrastrukturbetreiber zunehmend befinden, wird insofern problematisiert, als der damit verbundene Preisdruck Investitionen in „sicherheitsrelevante Vorkehrungen" (BMI 2011: 9) entgegenstehen könne (vgl. Folkers 2018a: 253). In der Konstellation aus staatlichen Stellen und vorwiegend privaten

8 Seit den 1960er-Jahren wurden Maßnahmen zur Aufrechterhaltung der Versorgung im Spannungs- oder Verteidigungsfall in Sicherstellungsgesetzen geregelt, die später durch Vorsorgegesetze für friedenszeitliche Versorgungskrisen ergänzt wurden (vgl. Kloepfer 2015: 196-210; BBK 2020: 45-47).
Betreibern wird der „institutionalisierten, organisierten Zusammenarbeit von Staat und Wirtschaft in etablierten Sicherheitspartnerschaften" (BMI 2009: 6) besondere Bedeutung beigemessen (vgl. auch BMI 2005: 6; BBK 2012: 24). Freiwilligen Maßnahmen wird Vorrang eingeräumt, gesetzgeberische Mittel aber ausdrücklich nicht ausgeschlossen (vgl. BMI 2009: 12-13)..$^{9}$ Der Staat, so heißt es im Leitbild der Strategie, steuere „primär moderierend, nötigenfalls normierend“ (BMI 2009: 2; vgl. BBK 2020: 22).

Welche Infrastrukturelemente als kritisch zu betrachten sind, wie also Kritikalität zu Zwecken der Identifizierung operationalisiert werden kann, blieb zunächst offen, wenn auch nicht unbearbeitet (vgl. Fekete 2011). Das 2015 verabschiedete IT-Sicherheitsgesetz (IT-SiG) enthält allerdings Vorgaben für die Betreiber kritischer Infrastrukturen ,im Sinne des Gesetzes‘ und zog daher die Etablierung eines rechtsverbindlichen, in der BSI-Kritisverordnung (BSI-KritisV) geregelten Identifizierungsverfahrens auf Bundesebene nach sich. ${ }^{10}$ Da Kritikalität als "relatives Maß" (BMI 2009: 5) aufgefasst wird, gilt auch die Identifizierung als kontextabhängiges Unterfangen, das heißt, ein Infrastrukturelement gilt nicht per se, sondern innerhalb eines abgesteckten Analyserahmens als kritisch (BBK 2020: 32-33). Um „Bedarfsträgern insbesondere in Ländern und Kommunen eine Anleitung für ihre eigene Identifizierung [zu] bieten" (BBK 2019b: 11), beschreibt die Arbeitshilfe "Identifizierung in sieben Schritten" (BBK 2019b) eine generische Version des in der BSI-Kritisverordnung implementierten Verfahrens.

Die sowohl der BSI-Kritisverordnung als auch der Arbeitshilfe zugrunde liegende Methode kombiniert ein qualitatives Kriterium, das die grundsätzliche Beteiligung eines Infrastrukturelements an der Bereitstellung einer kritischen Dienstleistung abbildet, mit einem quantitativen Kriterium, das sich auf den Beitrag eines Infrastrukturelements an der Bereitstellung einer kritischen Dienstleistung bezieht. Das qualitative Kriterium wird in der Verordnung abgedeckt, indem innerhalb der vom

9 Zur Rolle gesetzgeberischer Mittel beim Schutz kritischer Infrastrukturen vgl. Wiater (2013) und Wiater (2017); einen Überblick über die einschlägige Gesetzgebung des Bundes gibt BBK (2020: 41-43). 10 Eine 2008 verabschiedete EU-Richtlinie hatte bereits die Identifizierung europäischer kritischer Infrastrukturen, „deren Störung oder Zerstörung erhebliche Auswirkungen in mindestens zwei Mitgliedstaaten hätte" (Art. 2b RL 2008/114/EG), im Energie- und Verkehrssektor auf den Weg gebracht (vgl. § 12g EnWG; EnWG Energiewirtschaftsgesetz vom 7. Juli 2005, zuletzt geändert durch Artikel 1 des Gesetzes vom 5. Dezember 2019; vgl. RL 2008/114/EG - Richtlinie des Rates über die Ermittlung und Ausweisung europäischer kritischer Infrastrukturen und die Bewertung der Notwendigkeit, ihren Schutz zu verbessern vom 8. Dezember 2008). 
IT-Sicherheitsgesetz adressierten Sektoren ${ }^{11}$ kritische Dienstleistungen sowie Kategorien zu ihrer Bereitstellung erforderlicher Anlagen vorgegeben werden. ${ }^{12}$ Die Arbeitshilfe führt über mehrere Schritte zu einer kontextspezifischen Auswahl kritischer Dienstleistungen, aus der sich die an ihrer Bereitstellung beteiligten Infrastrukturelemente ableiten lassen (BBK 2019b: 31-41). Mit dem quantitativen Kriterium wird die Folgenschwere des Ausfalls eines Infrastrukturelements für die Verfügbarkeit einer kritischen Dienstleistung in den Blick genommen, genauer gesagt, die Anzahl der Menschen, die infolge dessen „direkt oder indirekt von einem Ausfall der kritischen Dienstleistung betroffen wäre" (BBK 2019b: 43). Die BSI-Kritisverordnung legt zu diesem Zweck einen Regelschwellenwert in der Höhe von 500.000 tatsächlich - oder rechnerisch von einem Infrastrukturelement - versorgten Personen zugrunde. ${ }^{13}$ Die Arbeitshilfe leitet dazu an, einen an der Einwohnerzahl des betrachteten Gebiets ausgerichteten Schwellenwert zu wählen: „Je größer die Region, desto höher sind im Allgemeinen die Schwellenwerte" (BBK 2019b: 44).

Wie verbreitet das in BBK (2019b) beschriebene Verfahren zur Identifizierung bereits angewendet wird, ist schwer zu sagen. Laut BBK (2020: 32) begleite man „derzeit einige Anwender bei der Umsetzung“. In der Praxis dürften teils individuell bedarfsangepasste Wege beschritten worden sein, um sich der Frage zu nähern, welche Infrastrukturelemente im konkreten Fall als kritisch zu betrachten sind, z. B. in der Einsatzplanung (vgl. Lange/Henke/Kariger 2015). Mit dem Landeskatastrophenschutzgesetz Mecklenburg-Vorpommern formuliert seit 2016 eine landesrechtliche Regelung explizit Vorgaben für die Betreiber kritischer Infrastrukturen (§ 13a LKatSG $M-V^{14}$ ), ein Identifizierungsverfahren wird dort allerdings nicht konkretisiert. Bisweilen ist nicht davon auszugehen, dass kritische Infrastrukturen außerhalb des vom IT-Sicherheitsgesetz definierten Rahmens bereits flächendeckend in einem formellen Verfahren identifiziert wurden. Allerdings verwenden viele der ab März 2020 im Zusammenhang mit der SARS-CoV2-Pandemie auf Länderebene erlassenen Regelungen den Begriff „kritische Infrastrukturen" und treffen, darauf Bezug nehmend, Festlegungen. ${ }^{15} \mathrm{Ob}$ und inwiefern

11 Vgl. Tabelle 1 mit Ausnahme der Sektoren „Kultur und Medien“ sowie "Staat und Verwaltung".

12 Vgl. Teil 1 Abs. 1 Anhänge 1-7 BSI-KritisV.

13 Vgl. „Berechnungsformeln zur Ermittlung der Schwellenwerte“ in Teil 2 der Anhänge 1-7 BSI-KritisV.

14 Landeskatastrophenschutzgesetz Mecklenburg-Vorpommern vom 15. Juli 2016, zuletzt geändert am 7. September 2016.

15 Vgl. https://www.bbk.bund.de/SharedDocs/Downloads/ dadurch längerfristig die formelle Identifizierung vorangetrieben wird, bleibt abzuwarten.

\section{Dem Schutz kritischer Infrastrukturen Rechnung tragen - Stand der Diskussion}

Wie dem Schutz kritischer Infrastrukturen bei raumbedeutsamen Planungen und Maßnahmen Rechnung getragen werden kann, ist zunächst ausgehend von einer Erläuterung in der Begründung zur Novellierung des Raumordnungsgesetzes thematisiert worden: ${ }^{16}$ „Eine parallele Trassenführung von verschiedenen Infrastrukturen", so heißt es dort (Deutscher Bundestag 2008: 21), sei „unter dem Aspekt des Schutzes kritischer Infrastrukturen sorgfältig zu prüfen“. Hintergrund ist der von Rinaldi, Peerenboom und Kelly (2001: 15) als "geographische Interdependenz" bezeichnete Umstand, dass mehrere unmittelbar nebeneinanderliegende Infrastrukturen demselben gefährlichen Ereignis ausgesetzt sein können (vgl. auch BBSR 2012: 54; Riegel 2014: 23-25). Die Trassenbündelung dient allerdings dazu, die „weitere Zerschneidung der freien Landschaft und von Waldflächen" zu vermeiden und die „Flächeninanspruchnahme im Freiland“ ( $\$ 2$ Abs. 2 Nr. 2 ROG) zu begrenzen (vgl. Riegel 2015a: 60-62).

Stehen sich unterschiedliche Belange in den Grundsätzen konflikthaft gegenüber, wie in der beschriebenen Konstellation, kommt es zu einem Abwägungsproblem (vgl. Runkel 2018: 2998). Es gilt zu entscheiden, in welchen Fällen die Interessen des Schutzes kritischer Infrastrukturen so hoch zu gewichten sind, dass ein Abweichen vom Bündelungsgebot gerechtfertigt wäre. Riegel (2015a: 127) nähert sich dem Problem von der Infrastrukturseite aus und schlägt einen Ansatz zur Ermittlung von Schwerpunkten „räumlicher Kritikalität“ als Entscheidungsgrundlage vor. Dort, wo sich durch Bündelung oder Kreuzung von Infrastrukturtrassen Schwerpunkte gebildet haben, könne mit Mitteln der Regionalplanung eine weitere Konzentration vermieden werden (Riegel 2015a: 121-167; Riegel 2015b: 268-276). Eine zu hohe Bündelung von Infrastrukturtrassen kann auch aus anderen Gründen, z. B. wegen des Immissionsschutzes

BBK/DE/Sonstiges/Covid-19_Kritis_Schulen_Kitas.pdf? blob=publicationFile (13.10.2020).

16 Einblicke in den Umgang mit dem zum Untersuchungszeitpunkt recht neuen Abwägungsbelang in der Praxis gibt Riegel (2014) sowie Riegel (2015a: 79-119). 
sowie des Natur- und Landschaftsschutzes oder aufgrund kumulativer Effekte als Überlastung des Raums, als Überbündelung (vgl. Pleiner 2016: 132; Pleiner 2018: 54; Scholles 2018: 39), abgelehnt werden. Es ist also durchaus denkbar, dass sich die Belange des Schutzes kritischer Infrastrukturen in Bezug auf die Trassenbündelung im konkreten Fall synergetisch zu weiteren Belangen verhalten - womit das oben genannte Abwägungsproblem komplexer ausfallen würde.

Unter der Annahme, dass sich die mit der Bündelung in Verbindung gebrachten Probleme dort verschärfen, wo das Auftreten bestimmter Ereignisse besonders wahrscheinlich ist, wird das Abwägungsproblem auch von der Gefahrenseite aus angegangen: Laut Handbuch zur Ausgestaltung der Hochwasservorsorge in der Raumordnung kann es im Interesse des Schutzes kritischer Infrastrukturen geboten sein, vom grundsätzlich geltenden Bündelungsprinzip abzuweichen, wenn „überschwemmungsgefährdete Bereiche tangiert sind" (BMVI 2017a: 39). Dass bestimmte Ereignisse in manchen Gebieten besonders wahrscheinlich auftreten, ist grundlegend für Ansätze der raumplanerischen Risikovorsorge. Eine räumlich differenzierte Eintrittswahrscheinlichkeit von Gefahrenereignissen und, damit verbunden, die Möglichkeit besonders gefährdete Gebiete abzugrenzen, gelten als Merkmale ,raumrelevanter Risiken' (in Abgrenzung zu mehr oder weniger ubiquitären Risiken; vgl. Greiving 2011: 22). Sofern die Eintrittswahrscheinlichkeit der fraglichen Ereignisse und/oder deren Folgen mit Instrumenten der räumlichen Planung beeinflusst werden können, werden sie zudem als „raumplanungsrelevant“ gesehen (Greiving 2011: 23). Diesem Ansatz folgend können kritische Infrastrukturen als „schutzwürdige Raumnutzung" betrachtet werden (Greiving/Hartz/Hurth et al. 2016: 94; Greiving 2018: 418). So verfährt das oben genannte Handbuch nicht nur, indem es auf mögliche Ausnahmen vom Bündelungsgebot hinweist, sondern auch, indem es dazu aufruft, Errichtung und Ausbau kritischer Infrastrukturen in überschwemmungsgefährdeten Bereichen möglichst zu vermeiden oder, falls unvermeidlich, Objektschutzmaßnahmen vorzuschreiben (BMVI 2017a: 39; vgl. auch Greiving 2018: 418). Exposition oder Verwundbarkeit des Schutzguts sollen also reduziert werden, um das Risiko zu mindern.

Als schutzwürdige Raumnutzung können kritische Infrastrukturen nicht nur im Hochwasserrisikomanagement, sondern generell in Verfahren zum Umgang mit raumplanungsrelevanten Risiken berücksichtigt werden. Diese Vorgehensweise wurde in einem Modellvorhaben zum vorsorgenden Risikomanagement in der Regionalplanung erprobt, das auch Risiken durch Erdbeben, Hitze und technische Störfälle betrachtete (vgl. Hartz/Saad/ Greiving et al. 2015; Greiving/Hartz/Hurth et al. 2016). Im Kontext der Störfallvorsorge - im engeren Sinne, dem Umgang mit Risiken im Umfeld von Anlagen, die der Störfall-Verordnung (12. BImSchV) $)^{17}$ unterliegen - könnten einige Infrastrukturelemente zudem eine Doppelrolle einnehmen: Es wird auf Konstellationen hingewiesen, in denen dieselbe Anlage sowohl im Kontext des Schutzes kritischer Infrastrukturen als auch im Kontext der Störfallvorsorge als relevant betrachtet und damit zum Gegenstand unterschiedlicher Risikomanagementansätze (mit spezifischen räumlichen Handlungslogiken) werden könnte (vgl. Krings 2018: 109-118). Auch jenseits des Geltungsbereichs der 12. BImSchV werden einige Infrastrukturen mit einem anlagenbezogenen Gefahrenpotenzial in Verbindung gebracht und nicht immer wurde und wird hinsichtlich dessen, was eine Infrastruktur zu einer kritischen Infrastruktur macht, klar zwischen „Versorgungswichtigkeit" und diversen Formen von „Gefährlichkeit“ unterschieden (vgl. Neisser 2016: 12-19; Krings 2018: 107-109).

Ausfälle kritischer Infrastrukturen werden nicht als raum- oder raumplanungsrelevante Gefahrenkategorie aufgefasst. Es sei zu erwarten, dass sich Ausfälle in Form von Domino- bzw. Kaskadeneffekten (vgl. Kapitel 2) ausbreiten, die, so BMVI (2017a: 39), „immer nur in szenariohaften Betrachtungen für umfassend definierte Einzelfälle ermittelt werden [können]“. Diese Szenarien zu analysieren sei nicht Aufgabe der Raumordnung, sondern des Katastrophenschutzes (BMVI 2017a: 39). Da sich entsprechende Ereignisse über große Distanzen und administrative Grenzen hinweg erstrecken können, komme es zudem zu einem Auseinanderklaffen von „Problemraum und Aufgabenwahrnehmungsraum" (BMVI 2017a: 38). Ausfallereignisse entziehen sich also gewissermaßen zweifach dem Zugriff der raumplanerischen Risikovorsorge. „Im Ergebnis“, so BMVI (2017a: 39), sei "der primäre Zugang der Raumordnung im Umgang mit kritischen Infrastrukturen der räumlich bestimmbare physische Standort der Infrastrukturen." Ein sachgerechter Umgang setze allerdings voraus, "dass dem Planungsträger bekannt ist, welche Infrastrukturen als kritisch einzuordnen [...] sind" (BMVI 2017a: 38; vgl. auch BBK 2020: 38) - eine Bedingung, die derzeit vielerorts nicht erfüllt sein dürfte (vgl. Kapitel 2). Sollte die formelle Identifizierung kritischer Infrastrukturen vorangetrieben

17 12. Verordnung zur Durchführung des Bundes-Immissionsschutzgesetzes (Störfall-Verordnung) in der Fassung der Bekanntmachung vom 15. März 2017, zuletzt geändert durch Artikel 1a der Verordnung vom 8. Dezember 2017. 
werden, könnte die Berücksichtigung der Belange des Schutzes kritischer Infrastrukturen für die räumliche Planung zukünftig erleichtert werden.

Der Raumordnungsbericht 2011 weist nicht nur auf Überschneidungen zwischen den Sektoren kritischer Infrastrukturen und Daseinsvorsorgebereichen hin, sondern bewertet unter der Überschrift „zentrale versus dezentrale Wasserversorgung" (BBSR 2012: 54) auch Varianten der räumlichen Organisation der Bereitstellung einer Daseinsvorsorgeleistung aus der Sicht des Schutzes kritischer Infrastrukturen. Ein Diskussionsstrang, der an etwaigen Überschneidungen zwischen Infrastrukturen, die als Mittel zur Bereitstellung von Daseinsvorsorgeleistungen Gegenstand politischer Entscheidungen und planerischer Praxis sind, und den als kritisch betrachteten Infrastrukturen ansetzt, hat sich allerdings bislang nicht herausgebildet. Entsprechende Überschneidungsbereiche werden in der Literatur verschiedentlich benannt (vgl. Engels/Nordmann 2018: 7; Krings 2018: 110) oder impliziert (vgl. Lauwe/Riegel 2008: 121), aber nicht zum Untersuchungsgegenstand gemacht. In rechts- und sozialwissenschaftlichen Arbeiten, die sich mit dem Beziehungsgefüge von „Daseinsvorsorge" und "kritische Infrastruktur" sowie den darauf bezogenen Politikbereichen befassen, werden räumliche oder raumordnungspolitische Aspekte nicht behandelt (Sonntag 2005: 116-136; Wiater 2013: 28-31) oder nur gestreift (Folkers 2017: 866-868; Folkers 2018a: 245-247; Folkers 2018b: 132). Diese Leerstelle soll der vorliegende Beitrag zu füllen beginnen. Anlass dazu gibt inzwischen auch die bereits genannte Arbeitshilfe zur Identifizierung: Kritische Dienstleistungen, so heißt es dort, seien „zumeist essentielle Leistungen der Daseinsvorsorge" (BBK 2019b: 17).

\section{Daseinsvorsorge: Gewährleistung von Versorgungsleistungen}

Der Begriff „Daseinsvorsorge“ geht auf den Juristen Ernst Forsthoff zurück, der in den 1930er-Jahren eine Diskrepanz zwischen Verwaltungsrecht und Verwaltungswirklichkeit beobachtete. ${ }^{18}$ Das am Modell des liberalen Rechtsstaats orientierte Verwaltungsrecht basiere auf der Annahme einer „prinzipiell unbegrenzten Sphäre

18 Zur Entwicklung, theoretischen und geschichtlichen Einordnung und Rezeption des Konzepts sowie zur Rolle Forsthoffs vgl. Scheidemann (1991) und Kersten (2005). individueller Freiheit und einer prinzipiell begrenzten Sphäre staatlichen Handelns" (Forsthoff 1938: 42). Ausgestattet mit grundrechtlich gesicherten Freiheiten habe jeder „für sein eigenes Wohl zu sorgen“, wobei vorausgesetzt werde, die "Zustände gestatten es inm auch, dafür sorgen zu können" (Forsthoff 1938: 44). Tatsächlich sei der Mensch aber „nicht mehr im Besitze der elementarsten Lebensgüter, ohne die sein physisches Dasein auch nicht einen Tag denkbar ist" (Forsthoff 1938: 7). Die Verwaltung trete daher mit der „Darbringung von Leistungen, auf welche der in die modernen massentümlichen Lebensformen verwiesene Mensch lebensnotwendig angewiesen ist" (Forsthoff 1938: 7), in einer existenziellen, aber rechtlich nicht vorgesehenen Funktion in Erscheinung. Diesem Missstand solle mit Schaffung einer Rechtsgrundlage für die von inm als "Daseinsvorsorge" bezeichneten Verwaltungstätigkeiten abgeholfen werden. ${ }^{19}$

Nach der Gründung der Bundesrepublik wurde Daseinsvorsorge zu einem prägenden politischen und verwaltungspraktischen Konzept. Dazu musste allerdings insbesondere sein Verhältnis zu den Grundrechten überdacht werden: Um im neuen Umfeld anschlussfähig zu sein, konnte die Daseinsvorsorge - anders als nach Forsthoffs (1938: 46) ursprünglicher Auffassung - nicht als Ersatz der Grundrechte verstanden werden. Liberale Werte und Wohlfahrtsstaatlichkeit mussten miteinander versöhnt werden (vgl. Folkers 2017: 866). „Wohlfahrtsstaat" bedeute, mit den Worten Baduras (1966: 625), „nicht Wohlfahrt statt Freiheit, sondern Freiheit durch Wohlfahrt" und es bestehe "die Chance der individuellen Selbstverwirklichung auf Grund der Wahrnehmung einer sozialen Verantwortung durch den Staat" (Hervorhebung im Original). Die Daseinsvorsorge soll die Grundrechte nicht länger ersetzen, sondern die Wahrnehmung grundrechtlich abgesicherter Freiheiten ermöglichen (vgl. Knauff 2004: 48).

Zudem widerspreche es, so Forsthoff (1959: 12) später entgegen seiner ursprünglichen Konzeption, „allen Anforderungen an den modernen Sozial- und Verteilungsstaat“, wolle man den Begriff „auf den der Vitalsphäre entnommenen Mindeststandard individueller Daseinsbehauptung beschränken“. Die „Versorgungsbedürfnisse des modernen Menschen“ seien „nicht von

19 Forsthoff (1938: 7) nennt an gleicher Stelle auch „die Gewährleistung eines angemessenen Verhältnisses von Lohn und Preis“ und "die Lenkung des Bedarfs, der Erzeugung und des Umsatzes“ als Aspekte der Daseinsvorsorge, führt allerdings nicht dazu aus. Sie spielen in späteren Diskussionen um die Daseinsvorsorge keine Rolle. 
dem allgemeinen Lebensstandard zu lösen und damit nicht konstant" (Forsthoff 1959: 12). Es ist also konzeptionell gedeckt, die Bandbreite der Daseinsvorsorge zugerechneter Leistungen im Zuge der wohlfahrtsstaatlichen Entwicklung der folgenden Jahrzehnte zu erweitern. Daseinsvorsorge soll nicht länger das ,nackte Leben' ermöglichen, sondern ein ,gutes Leben' (Folkers 2018b: 132). Im Umfeld eines „allgemeinen Ausbaus des Sozialstaats“ kommt es zu einer „Ausweitung des daseinsvorsorgerischen Leistungsspektrums", was sich mit zunehmender Anerkennung von „Daseinsvorsorge als Staatsaufgabe“ in einem "starken Anstieg der leistenden Staatstätigkeit“" (Knauff 2004: 54) niederschlägt.

Diese Entwicklungen bedingen einen erheblichen Ausbau jener Arrangements, für die sich zeitgleich der Begriff "Infrastruktur" etablierte. Im Kontext eines "ständig steigenden und von gleichsam unsichtbaren ,öffentlichen Händen' bereitgestellten, sozial- und interventionsstaatlichen Betreuungsniveaus" (van Laak 1999: 299) tritt der in den 1950er-Jahren aus dem NATOVokabular in den deutschen Sprachgebrauch übernommene Begriff seine Karriere an. Er erfreut sich schnell großer Beliebtheit und entwickelt sich zu einer "handlichen, weil scheinbar unpolitisch-technischen Kategorie für staatliche Vorleistungen im Wirtschaftlichen wie im Sozialen“ (van Laak 1999: 285). "Infrastruktur" wird zum technokratisch-neutral anmutenden Pendant des Konzepts "Daseinsvorsorge“, wobei deren Abgrenzung bis heute zuweilen schwammig ausfällt und die Präferenz für den einen oder anderen Begriff auch konjunkturellen Schwankungen unterliegt (vgl. Schmidt 2013: 15-28; Steinführer 2015: 7).

In den 1990er-Jahren wird mit der Konturierung des Gewährleistungsstaates die Frage nach der Reichweite staatlicher Verantwortung für die Daseinsvorsorge gestellt. Dem vorangegangen war seit den 1970er-Jahren ein erst schleichend und überlagert von einem parallel weitergehenden Ausbau öffentlichen Engagements vollzogener ,faktischer Paradigmenwechsel' (Knauff 2004: 58): von der ganz überwiegenden Leistungserbringung durch öffentliche hin zur vielfältigen Einbindung privater Akteure. Eingebettet in eine Programmatik zur Verschlankung des Staats (z. B. Deutscher Bundestag 1998) und flankiert von europarechtlichen Impulsen (z. B. KOM 1996) wird hinsichtlich der staatlichen Verantwortung für die Daseinsvorsorge nun verstärkt zwischen der Gewährleistung (ob) und den Modalitäten ihrer Erfüllung (wie) unterschieden (vgl. Knauff 2004: 66): Beschränkt sich die staatliche Verantwortung für eine Aufgabe auf die Gewährleistung, so kann die eigentliche Erfüllung durch unterschiedliche Akteure erfolgen. Die öffentliche
Hand verzichtet bei der Leistungserbringung vielfach auf ihre „bevorzugte Stellung und wird zum ,normalen Marktteilnehmer" (Knauff 2004: 67) neben privaten, privatisierten oder öffentlich-privaten Akteuren.

Vor diesem Hintergrund entwickelt sich ab Mitte der 1990er-Jahre eine Debatte um „das nach juristischen und politischen Maßstäben richtige Maß der Staatsintervention“, die zu einer „Rückbesinnung auf die Bedeutung öffentlicher Versorgung" (Milstein 2018: 364) führt und den Begriff "Daseinsvorsorge“ in den akademischen und politischen Diskurs zurückbringt (Krajewski 2011: 29). Die seit den 2000er-Jahren in einigen Bereichen der Daseinsvorsorge zu beobachtenden Rekommunalisierungen (vgl. Libbe 2012; Cumbers/Becker 2018) bilden eine Art Gegentrend im Bereich der Erfüllung, die hier skizzierte Verantwortungsteilung stellen sie aber nicht automatisch infrage. „Der Begriff Daseinsvorsorge“, so lässt sich bisweilen definieren, „umfasst gemeinwohldienliche Leistungen im weiteren Sinn, die der Einzelne zu einer angemessenen Lebensführung benötigt und deren grundsätzlich marktförmige Darbietung daher regelmäßig staatlichem Einfluss unterliegt" (Milstein 2018: 361). Um welche Leistungen es sich dabei im Einzelnen handelt, „ist grundsätzlich zeitbezogen politisch zu beantworten" (Knauff 2004: 49). ${ }^{20}$

\section{Sektoren kritischer Infrastrukturen und Bereiche der Daseinsvorsorge}

In den Konzepten „Daseinsvorsorge“ und „kritische Infrastruktur" kommt auf jeweils unterschiedliche Weise die Anerkennung gesellschaftlicher Relevanz und staatlicher Verantwortung für bestimmte Versorgungsleistungen zum Ausdruck (vgl. Kapitel 2 und Kapitel 4). Dabei nimmt die Bedeutung von Versorgung und den Einrichtungen des Versorgens eine jeweils spezifische Form an: Ihre Verfügbarkeit wird im Kontext der Daseinsvorsorge als Grundvoraussetzung der individuellen Lebensführung und des sozialen Gefüges konzeptualisiert, ihre NichtVerfügbarkeit tritt beim Schutz kritischer Infrastrukturen als existenzielle Gefahr in Erscheinung. Dabei kommt in der Idee der kritischen Infrastrukturen eine ambiva-

20 Die Interpretation des Leistungsspektrums der Daseinsvorsorge ist damit ein prinzipiell unabgeschlossener Prozess, dessen Fortgang sich jüngst anhand der Bereitstellung von (Breitband-)Internetanbindung beobachten lässt (z. B. WD 2012; Beirat für Raumentwicklung 2015). 
lente Haltung gegenüber einem mit Werten wie „soziale Sicherheit, staatliche Verantwortung und wirtschaftliche Prosperität" (van Laak 2001: 367) konnotierten und mit "gesellschaftliche[r] Kohäsion“ (Kaufmann 2011: 107) assoziierten Begriff zum Ausdruck: „Nicht mehr nur der Mangel an Infrastruktur", sondern auch deren Verdichtung, Vernetzung und "gar der reibungslose Normalbetrieb der Systeme" (Kaufmann 2011: 107) gelten nun als Problem. Während im Kontext der Daseinsvorsorge das ermöglichende Element mittels Infrastrukturen bereitgestellter Versorgungsleistungen im Mittelpunkt steht, rückt mit dem Schutz kritischer Infrastrukturen der Aspekt der Gefahrenabwehr in den Vordergrund.

Überschneidungen der Gegenstandsbereiche von Daseinsvorsorge und Schutz kritischer Infrastrukturen erscheinen intuitiv naheliegend. Mangels einer verbindlichen Liste von Bereichen der Daseinsvorsorge entzieht sich die Aussage, diese könnten mehrheitlich einem Sektor oder Teilsektor kritischer Infrastrukturen zugeordnet werden (BBSR 2012: 53), allerdings der unmittelbaren Überprüfung. In behördlichen Veröffentlichungen werden, sofern sie denn eine Liste von Bereichen der Daseinsvorsorge enthalten, regelmäßig Formulierungen gewählt, die einen Anspruch auf Vollständigkeit zerstreuen. So mutet die in Tabelle 2 in Spalte 2 enthaltene Liste von „Infrastrukturen der Daseinsvorsorge“ (BMVBS 2010: 36) zwar umfänglich an, wird im begleitenden Text aber als nicht abschließend bezeichnet. Die juristische Konkretisierung des Leistungsumfangs der Daseinsvorsorge erfolgt sukzessive durch die Rechtsprechung. Spalte 3 enthält die von Krajewski (2011: 32-35) und Milstein (2018: 365-367) vorgestellte Kasuistik. Der Fachliteratur sind darüber hinaus auf der Basis unterschiedlicher Kriterien erstellte, mehr oder weniger stark voneinander abweichende Auflistungen zu entnehmen, etwa die von Einig (2008: 18) vorgeschlagene Variante (vgl. Spalte 4). Oft werden - wie in Tabelle 2 - mehrere Versionen vergleichend gegenübergestellt, um der Vielfalt unterschiedlicher Auffassungen Rechnung zu tragen (vgl. Steinführer 2015: 7; BMVI 2017b: 17-19).

Die Einträge in den Spalten 2-4 weichen hinsichtlich inres Abstraktionsgrades voneinander ab, bezeichnen zum Teil Einrichtungstypen, zum Teil Dienstleistungen und repräsentieren erwartungsgemäß unterschiedliche Auffassungen davon, was zur Daseinsvorsorge zählt. Einige Bereiche tauchen in vergleichbarer Weise in allen drei Spalten auf (z. B. Kinderbetreuung), es scheint also eine Art gemeinsamen Nenner zu geben, andere kommen nur einmal vor (z. B. Deichbau). Tatsächlich haben einige der übereinstimmend genannten Bereiche auch ein Pendant unter den Sektoren kritischer Infra- strukturen, wobei man die Branchen hinzuziehen muss, um das Notfall- und Rettungswesen im Sektor „Staat und Verwaltung" ausfindig zu machen. Nicht in allen Fällen ist klar, ob partielle Überschneidungen bestehen könnten (z.B. zwischen Pflegebereich und Gesundheitssektor). Einige soziale Dienstleistungen wie Kinderbetreuung und Bildung, aber auch der eher technische Bereich der Abfallentsorgung werden einhellig zur Daseinsvorsorge gezählt, haben allerdings keine noch so grobe Entsprechung unter den Sektoren kritischer Infrastrukturen. Umgekehrt hat der Sektor „Ernährung“ kein Gegenüber in den Spalten 2-4. Ein Blick auf die Branchen verrät, dass auch andere Teilsektoren kritischer Infrastrukturen nicht in den hier genannten Daseinsvorsorgebereichen repräsentiert sind.

Den für Tabelle 2 herangezogenen Quellen nach sind weder die Sektoren kritischer Infrastrukturen sämtlich eine Teilmenge der Bereiche der Daseinsvorsorge, noch sind alle Daseinsvorsorgebereiche in den Sektoren wiederzufinden. Für viele Bereiche der Daseinsvorsorge kann aber zumindest von einer gewissen Vergleichbarkeit mit einem Sektor gesprochen werden. Ob das auf die meisten Bereiche zutrifft (vgl. BBSR 2012: 53), hängt davon ab, welcher Auffassung vom Spektrum der Daseinsvorsorge man folgt. Umgekehrt lassen viele Sektoren kritischer Infrastrukturen Parallelen zu einem Daseinsvorsorgebereich erkennen.

Bei einem genaueren Blick in die Arbeitshilfe zur Identifizierung kritischer Infrastrukturen (BBK 2019b) zeigt sich allerdings, dass die Sektoreneinteilung zumindest unterhalb der Bundesebene keine Ausschlusswirkung entfaltet: Die Arbeitshilfe leitet explizit dazu an, auch Infrastrukturelemente aus Daseinsvorsorgebereichen, die keine Überschneidungen auf der Ebene der Sektoren aufweisen, bei der Identifizierung zu berücksichtigen. So sei es beim Schritt „Erhebung der Dienstleistungen“ besonders für Kommunen sinnvoll, „Leistungen aus dem Bereich der Daseinsvorsorge zu ergänzen, welche noch nicht über die Sektoren und Branchen KRITIS [kritischer Infrastrukturen] erfasst werden“ (BBK 2019b: 32; meine Hervorhebung). Folglich können die auf diesem Weg identifizierten kritischen Infrastrukturen auch aus Daseinsvorsorgebereichen ohne Pendant unter den Sektoren kritischer Infrastrukturen stammen. Die Gegenstandsbereiche von Daseinsvorsorge und Schutz kritischer Infrastrukturen könnten sich demnach vor allem in der kommunalen Praxis noch weiter überschneiden, als Tabelle 2 vermuten lässt.

Dass die Bandbreite der Dienstleistungen, die unterhalb der Bundesebene als kritisch betrachtet werden, deutlich über die Sektoreneinteilung hinausgehen kann, 
Tabelle 2: Sektoren und Branchen kritischer Infrastrukturen und Bereiche der Daseinsvorsorge ${ }^{a}$

\begin{tabular}{llll}
\hline $\begin{array}{l}\text { Sektoren und Branchen } \\
\text { kritischer Infrastrukturen } \\
\text { (BBK 2020: 24) }\end{array}$ & $\begin{array}{l}\text { „Infrastrukturen der } \\
\text { Daseinsvorsorge“ } \\
\text { (BMVBS 2010: 36) }\end{array}$ & $\begin{array}{l}\text { „Umfang der } \\
\text { Daseinsvorsorge“ } \\
\text { (Krajewski 2011: 32-35; } \\
\text { Milstein 2018: 365-367) }\end{array}$ & $\begin{array}{l}\text { "Aufgabenfelder } \\
\text { offentlicher } \\
\text { Daseinsvorsorge“ } \\
\text { (Einig 2008: 18) }\end{array}$ \\
\hline $\begin{array}{l}\text { Energie } \\
\text { Elektrizität, Mineralöl, Gas }\end{array}$ & $\begin{array}{l}\text { Gasversorgung, } \\
\text { Fernwärmeversorgung, } \\
\text { Stromversorgung }\end{array}$ & $\begin{array}{l}\text { Energieversorgung (Gas, } \\
\text { Elektrizität, Fernwärme) }\end{array}$ & Energieversorgung \\
\hline
\end{tabular}

\section{Ernährung}

Ernährungswirtschaft,

Lebensmittelhandel

\begin{tabular}{|c|c|c|c|}
\hline $\begin{array}{l}\text { Finanz- und Versicherungswesen } \\
\text { Banken, Börsen, Versicherungen, } \\
\text { Finanzdienstleister }\end{array}$ & & $\begin{array}{l}\text { öffentlich-rechtliche } \\
\text { Finanzinstitutionen (z. B. } \\
\text { Sparkassen, Landesbanken) }\end{array}$ & $\begin{array}{l}\text { Finanz- und Versicherungs- } \\
\text { dienstleistungen }\end{array}$ \\
\hline $\begin{array}{l}\text { Gesundheit } \\
\text { medizinische Versorgung, } \\
\text { Arzneimittel und Impfstoffe, Labore }\end{array}$ & $\begin{array}{l}\text { Hausärztliche Versorgung, } \\
\text { Krankenhäuser }\end{array}$ & $\begin{array}{l}\text { Gesundheitsleistungen } \\
\text { (Krankenhäuser, } \\
\text { Krankenkassen, Vertragsärzte) }\end{array}$ & Gesundheitswesen \\
\hline $\begin{array}{l}\text { Informationstechnik und } \\
\text { Telekommunikation } \\
\text { Telekommunikation und } \\
\text { Informationstechnik }\end{array}$ & $\begin{array}{l}\text { Informations- und } \\
\text { Kommunikationssysteme } \\
\text { (Grundversorgung, höhere } \\
\text { Leistungsbandbreiten) }\end{array}$ & Telekommunikation & $\begin{array}{l}\text { Kommunikationsdienstlei- } \\
\text { stungen }\end{array}$ \\
\hline $\begin{array}{l}\text { Medien und Kultur } \\
\text { Rundfunk (Fernsehen, Radio), } \\
\text { gedruckte und elektronische Presse, } \\
\text { Kulturgut, symbolträchtige Bauwerke }\end{array}$ & $\begin{array}{l}\text { Kunst- und Kulturpflege, } \\
\text { Bibliotheken }\end{array}$ & $\begin{array}{l}\text { Rundfunk, Kultureinrichtungen } \\
\text { (Stadthallen, Theaterbetriebe) }{ }^{\mathrm{b}}\end{array}$ & Kulturelle Versorgung \\
\hline $\begin{array}{l}\text { Staat und Verwaltung } \\
\text { Regierung und Verwaltung, } \\
\text { Parlament, Justizeinrichtungen, } \\
\text { Notfall- und Rettungswesen } \\
\text { einschließlich Katastrophenschutz }\end{array}$ & $\begin{array}{l}\text { Polizei, Brandschutz, } \\
\text { Katastrophenschutz, } \\
\text { Rettungsdienst }\end{array}$ & Notfalltransporte & $\begin{array}{l}\text { Katastrophenschutz, } \\
\text { Feuerwehr und } \\
\text { Rettungswesen }\end{array}$ \\
\hline $\begin{array}{l}\text { Transport und Verkehr } \\
\text { Luftfahrt, Seeschifffahrt, Binnen- } \\
\text { schifffahrt, Schienenverkehr, } \\
\text { Straßenverkehr, Logistik }\end{array}$ & $\begin{array}{l}\text { Straßen (vornehmlich } \\
\text { Erschließungsfunktion und } \\
\text { übergeordnetes Netz), ÖPNV } \\
\text { und Schülertransport }\end{array}$ & $\begin{array}{l}\text { Verkehr (Verkehrsinfrastruktur, } \\
\text { Verkehrsdienstleistungen, } \\
\text { insbesondere ÖPNV), Post }\end{array}$ & $\begin{array}{l}\text { Verkehrsinfrastruktur, } \\
\text { Verkehrsdienste (wie } \\
\text { Schülertransport und } \\
\text { Öffentlicher Verkehr) }\end{array}$ \\
\hline \multirow[t]{7}{*}{$\begin{array}{l}\text { Wasser } \\
\text { öffentliche Wasserversorgung und } \\
\text { öffentliche Abwasserbeseitigung }\end{array}$} & $\begin{array}{l}\text { Trinkwasserversorgung, } \\
\text { Schmutz- und } \\
\text { Regenwasserkanalisation }\end{array}$ & $\begin{array}{l}\text { Trinkwasser, } \\
\text { Abwasserbeseitigung }\end{array}$ & $\begin{array}{l}\text { Wasserwirtschaft } \\
\text { (einschließlich Ver- und } \\
\text { Entsorgung) }\end{array}$ \\
\hline & $\begin{array}{l}\text { Mobile und stationäre } \\
\text { Pflegeeinrichtungen, } \\
\text { Begegnungsstätten für ältere } \\
\text { Menschen }\end{array}$ & Alten- und Pflegeheime & Altenpflege \\
\hline & $\begin{array}{l}\text { Jugendzentren, } \\
\text { Kindertageseinrichtungen }\end{array}$ & Kindergärten & Kinderbetreuung \\
\hline & $\begin{array}{l}\text { Grundschulen, allgemein- } \\
\text { und berufsbildende Schulen, } \\
\text { Volkshochschulen }\end{array}$ & Öffentliche Schulen & Schule und Bildungswesen \\
\hline & Abfallentsorgung & Abfallbeseitigung & Abfallwirtschaft \\
\hline & Sportstätten & $\begin{array}{l}\text { Sporteinrichtungen (Sportplätze, } \\
\text { Schwimmbäder) }\end{array}$ & Sportstätten \\
\hline & Friedhöfe & & Friedhöfe \\
\hline
\end{tabular}


Tabelle 2: Sektoren (und Branchen) kritischer Infrastrukturen und Bereiche der Daseinsvorsorge ${ }^{a}$ Continued

\begin{tabular}{|c|c|c|c|}
\hline \multirow[t]{7}{*}{$\begin{array}{l}\text { Sektoren und Branchen } \\
\text { kritischer Infrastrukturen } \\
\text { (BBK 2020: 24) }\end{array}$} & $\begin{array}{l}\text { „Infrastrukturen der } \\
\text { Daseinsvorsorge“" } \\
\text { (BMVBS 2010: } 36 \text { ) }\end{array}$ & $\begin{array}{l}\text { "Umfang der } \\
\text { Daseinsvorsorge“" } \\
\text { (Krajewski 2011: 32-35; } \\
\text { Milstein 2018: 365-367) } \\
\end{array}$ & $\begin{array}{l}\text { „Aufgabenfelder } \\
\text { öffentlicher } \\
\text { Daseinsvorsorge“ } \\
\text { (Einig 2008: 18) } \\
\end{array}$ \\
\hline & Kirchen & & \\
\hline & $\begin{array}{l}\text { Service- und Beratungsstellen } \\
\text { (Familienförderung, } \\
\text { Jugendämter, soziale } \\
\text { Sicherungssysteme) }\end{array}$ & Sozialhilfe & \\
\hline & $\begin{array}{l}\text { Einrichtungen für Obdach- und } \\
\text { Wohnungslose }\end{array}$ & & \\
\hline & Naherholung & & \\
\hline & & sozialer Wohnungsbau & $\begin{array}{l}\text { Wohnungswirtschaft } \\
\text { (sozialer Wohnungsbau) }\end{array}$ \\
\hline & & & Deichbau \\
\hline
\end{tabular}

\footnotetext{
${ }^{a}$ Anordnung aller Einträge orientiert sich an den alphabetisch angeordneten Sektoren kritischer Infrastrukturen. Formulierungen wurden vorzugsweise wörtlich übernommen, aber in Einzelfällen minimal abgekürzt. Nennungen wurden teilweise zu einem Eintrag zusammengefasst bzw. aufgespalten.

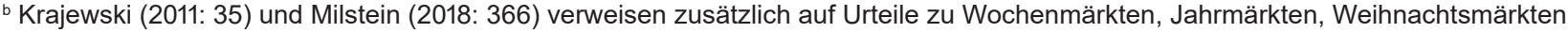
und Volksfesten, die durch ihren Veranstaltungscharakter etwas herausstechen und (mit einem gewissen Anteil Nahversorgung) wohl am ehesten als Formen der Brauchtumspflege dem Kulturbereich zugerechnet werden können.
}

zeigt sich zudem aktuell im Zusammenhang mit der SARS-CoV-2-Pandemie. In den zur Bewältigung der Lage länderseitig erlassenen Vorgaben wird das Spektrum kontextspezifisch ausgelegt und dabei teilweise deutlich erweitert. So enthalten beispielsweise die im März 2020 beschlossenen „Leitlinien zur Bestimmung des Personals kritischer Infrastrukturen" der Landesregierung Nordrhein-Westfalen einen zehnten Sektor, der Bezüge zu weiteren, in Tabelle 2 genannten Daseinsvorsorgebereichen erkennen lässt: „Schulen, Kinder- und Jugendhilfe, Behindertenhilfe". ${ }^{21}$ Es wird erst im Nachgang zu untersuchen sein, inwieweit die Erfahrung der SARS-CoV-2-Pandemie das Verständnis von kritischen Infrastrukturen auch längerfristig verändern. Vorerst ist festzuhalten, dass die Sektoreneinteilung unterhalb des Bundes nicht als einschränkende Vorgabe gelten kann.

Die Gegenüberstellung von Sektoren kritischer Infrastrukturen und Daseinsvorsorgebereichen (vgl. Tabelle 2) stützt die Vermutung, dass die kritischen Dienstleistungen, deren Ausfall im Kontext des Schutzes kritischer Infrastrukturen adressiert wird, zu einem erheblichen Anteil als Leistungen der Daseinsvorsorge bereitge-

$21 \mathrm{https}: / / w w w . l a n d . n r w / d e / p r e s s e m i t t e i l u n g / n e u e-l e i t l i n i e-b e-$ stimmt-personal-kritischer-infrastrukturen (13.10.2020). stellt werden. Zudem ist die Bindungswirkung der Sektorenliste unterhalb der Bundesebene begrenzt, sodass auch Dienstleistungen aus Daseinsvorsorgebereichen als kritisch betrachtet werden können, die sich keinem Sektor zuordnen lassen (vgl. BBK 2019b: 32). Der Versorgungsnormalzustand, vor dessen Hintergrund sich der Versorgungsausfall als Ausnahmesituation abhebt, dürfte maßgeblich von der Bereitstellung von Leistungen der Daseinsvorsorge geprägt sein und viele als kritisch bewertete Infrastrukturen dürften zugleich als Infrastrukturen der Daseinsvorsorge behandelt werden. Diese ,kritischen Infrastrukturen der Daseinsvorsorge' werden im doppelten Sinne als versorgungsrelevant betrachtet und sind in beiderlei Hinsicht Gegenstand von Entscheidungen und Maßnahmen - einschließlich der damit gegebenenfalls verbundenen Wechselwirkungen.

Dass sich Politik und Praxis der Daseinsvorsorge auf die Rahmenbedingungen des Schutzes kritischer Infrastrukturen auswirken, lässt sich beispielsweise anhand der Betrachtung der betreffenden Infrastrukturen „unter Aspekten der Eigentumsverhältnisse“ (BMI 2009: 6) nachvollziehen. Diese, so heißt es in der „Nationalen Strategie zum Schutz Kritischer Infrastrukturen“, würden mehrheitlich von „privaten, zum Teil erst kürzlich privatisierten Unternehmen betrieben und gesteuert" 
(BMI 2009: 6; vgl. Kapitel 2), dürften jedoch nicht als kritische Infrastrukturen, sondern vielfach als Infrastrukturen der Daseinsvorsorge in privaten Besitz übergegangen sein: Die für den Schutz kritischer Infrastrukturen charakteristische Akteurkonstellation aus staatlichen Stellen und mehrheitlich privaten Betreibern ergibt sich auch aus einer am Modell des Gewährleistungsstaats ausgerichteten Daseinsvorsorge (vgl. Kapitel 4). Der „Trend“, durch den „die Verantwortung für die Sicherheit, Zuverlässigkeit und Verfügbarkeit zunehmend in private, zumindest aber geteilte Verantwortung [übergeht]" (BMI 2009: 6), ist daseinsvorsorgepolitisches Programm (vgl. Wiater 2017: 231; Folkers 2018a: 250).

\section{Einladung zum Perspektivwechsel}

Es liegt nahe, dass auch die räumliche Planung gewissermaßen ,über den Umweg der Daseinsvorsorge;, genauer gesagt, über die räumliche Organisation der Bereitstellung von Daseinsvorsorgeleistungen, die Bedingungen für den Schutz kritischer Infrastrukturen mitgestaltet. Diese Vermutung lädt zu einem Perspektivwechsel ein, zur Betrachtung von Zielvorstellungen und Instrumenten der räumlichen Planung aus dem Blickwinkel des Schutzes kritischer Infrastrukturen. Da Versorgung beim Schutz kritischer Infrastrukturen auch die Gefahr des Versorgungsausfalls in sich trägt und die kritische Infrastruktur als Schutzgut und Gefahrenquelle zwei Rollen spielt (vgl. Kapitel 2), eröffnet dieser Perspektivwechsel allerdings mitunter irritierende Ansichten, wie sich anhand der Zielsetzung gleichwertiger Lebensverhältnisse veranschaulichen lässt.

Die raumordnungspolitische Deutung des Begriffs Daseinsvorsorge ist eng mit dieser aus Art. 72 Abs. 2 $\mathrm{GG}^{22}$ abgeleiteten und in $\S 1 \mathrm{Abs}$. 2 ROG verankerten Zielsetzung verbunden, wobei „die Daseinsvorsorge mit ihren unterschiedlichen Bereichen [...] als eine wesentliche Voraussetzung für gleichwertige Lebensverhältnisse [gilt]“" (BBSR 2017: 6). Den Grundsätzen der Raumordnung nach ist „die Versorgung mit Dienstleistungen und Infrastrukturen der Daseinsvorsorge, insbesondere die Erreichbarkeit von Einrichtungen und Angeboten der Grundversorgung für alle Bevölkerungsgruppen, zur

22 Grundgesetz für die Bundesrepublik Deutschland in der im Bundesgesetzblatt Teil III, Gliederungsnummer 100-1, veröffentlichten bereinigten Fassung, zuletzt geändert durch Artikel 1 des Gesetzes vom 15. November 2019.
Sicherung der Chancengleichheit in den Teilräumen in angemessener Weise zu gewährleisten" (§ 2 Abs. 2 Nr. 3 ROG). Die Verknüpfung mit dem Ziel gleichwertiger Lebensverhältnisse verleiht dem Anspruch, jede Einzelne und jeden Einzelnen an den Leistungen der Daseinsvorsorge teilhaben zu lassen (vgl. Kapitel 4), eine raumordnungspolitische Dimension. Nach aktueller Auslegung gilt das Gleichwertigkeitspostulat in Bezug auf die Daseinsvorsorge als erfüllt, „wenn in den Teilräumen des Bundesgebietes eine vergleichbare Mindestversorgung erreicht ist", wenn die Leistungen der Daseinsvorsorge „in einer akzeptablen Mindestqualität, zu sozialverträglichen Preisen und in befriedigender Zugänglichkeit (Erreichbarkeit) angeboten werden" (BBSR 2012: 31; vgl. auch BBSR 2017: 6-7). ${ }^{23}$ Die räumliche Differenzierung im Daseinsvorsorgeangebot soll sich also, im Sinne einer "Sockelgleichwertigkeit" (BBSR 2012: 16), oberhalb eines bestimmten Niveaus bewegen.

Für eine Betrachtung aus der Perspektive des Schutzes kritischer Infrastrukturen bieten sich gleich mehrere Anknüpfungspunkte an. Durch das „unentrinnbare Nebeneinander von Ermöglichung und Gefährdung" (Engels/Nordmann 2018: 7), das dem Konzept der Kritikalität innewohnt, setzt etwa eine möglichst universelle Teilhabe an einer Versorgungsleistung auch Jede und Jeden der Gefahr ihres Ausfalls aus (vgl. Krings 2018: 110). Die Verknüpfung von "Daseinsvorsorge" und "gleichwertigen Lebensverhältnissen“ verleiht diesem Dilemma eine räumliche Dimension: Der kritischen Dienstleistung wohnt die Gefahr ihres Ausfalls inne und zwar überall dort, wo sie bereitsteht. Ein Hinwirken auf eine räumlich ausgeglichene Verfügbarkeit der betreffenden Dienstleistungen begünstigt demnach eine Versorgungssituation, die Ausfälle als quasi ubiquitäre, nur in Form von Szenarien räumlich dingfest zu machende Gefahrenart erscheinen lässt (vgl. Kapitel 3). Allerdings können Anforderungen an die Erreichbarkeit von Einrichtungen der Daseinsvorsorge im Sinne gleichwertiger Lebensverhältnisse zudem mit der Entstehung redundanter Strukturen im Raum in Verbindung gebracht werden. "Redundanz" bezeichnet „das mehrfache Vorhandensein von Strukturen zur Erbringung derselben Leistung" (Lenz 2009: 58) und gilt im Kontext des Schutzes kritischer Infrastrukturen als wichtiger Faktor für die Bewältigung von Ausfallereignissen: Gelingt es, über redundante Strukturen den Ausfall einzelner Inf-

23 Zur Diskussion über Auslegung und Stellenwert der Zielsetzung seit den 1970er-Jahren vgl. BMVBS/BBSR (2009: 11-21); zur fachöffentlichen Debatte der letzten Jahre z. B. Klingholz (2015), ARL (2016) und Terfrüchte (2019). 
rastrukturelemente ganz oder teilweise zu kompensieren, kann die Verfügbarkeit der kritischen Dienstleistung zumindest eingeschränkt oder für einen gewissen Zeitraum aufrechterhalten werden (Lenz 2009: 58-59; BMI 2011: 19). Insofern kann sich eine im Interesse ihrer Erreichbarkeit dezentral organisierte Bereitstellung von Daseinsvorsorgeleistungen gleichzeitig förderlich auf die Voraussetzungen zum Umgang mit Ausfällen kritischer Infrastrukturen auswirken.

Es wäre widersinnig, den Schutz vor dem Ausfall einer Dienstleistung mit dem vorsorglichen Verzicht auf ihre Bereitstellung zu erkaufen: Dass Daseinsvorsorgeleistungen allen zur Verfügung stehen sollen, wird durch die Möglichkeit ihres Ausfalls nicht grundsätzlich infrage gestellt. Daher ist die Einsicht, dass ein flächendeckend vergleichbares Mindestangebot an Versorgungsleistungen unweigerlich mit einer vergleichbaren ,Mindestfallhöhe' möglicher Versorgungsausfälle korrespondiert, zwar grundsätzlich, ihr praktischer Nutzen dürfte aber ausgesprochen begrenzt sein. Redundante Strukturen als Ressource zu begreifen, könnte hingegen durchaus eine Neubewertung des vorgefundenen Infrastrukturbestands oder von Planungsalternativen nach sich ziehen. In diesem Fall lenkt der Perspektivwechsel die Aufmerksamkeit darauf, wie die Versorgung mit Leistungen der Daseinsvorsorge im Sinne gleichwertiger Lebensverhältnisse räumlich organisiert wird. Dieselbe Vorgehensweise kann in der Auseinandersetzung mit Ansätzen der räumlichen Daseinsvorsorgeplanung, wie der Konzentration von Einrichtungen der Daseinsvorsorge in Zentralen Orten (Kapitel 6.1) oder der Zentralisierung der Bereitstellung von Daseinsvorsorgeleistungen (Kapitel 6.2), den Blick für deren mögliche Implikationen für den Schutz kritischer Infrastrukturen schärfen und durchaus planungspraktische Fragen aufwerfen.

\subsection{Kritische Infrastrukturen in Zentralen Orten?}

Zu den prominentesten Planungsinstrumenten gehört das bereits seit den 1960er-Jahren umfassend implementierte Zentrale-Orte-Konzept (ZOK; vgl. Blotevogel 1996; Terfrüchte/Flex 2018), „mit dem überörtlich die Leistungserbringung der Daseinsvorsorge in Infrastruktureinrichtungen räumlich organisiert wird" (Pütz/Spangenberg 2006: 337). In den länderspezifischen Varianten des Zentrale-Orte-Konzepts werden typischerweise mehrere Hierarchiestufen Zentraler Orte mit charakteristischen Ausstattungsmerkmalen sowie Erwägungen zu Tragfähigkeit und Erreichbarkeit ver- knüpft (vgl. Greiving/Flex/Terfrüchte 2015; BBSR 2017: 34-47). Auf dieser Grundlage machen landesweite und regionale Raumordnungspläne Zentrale Orte zu „Normadressat[en] der zentralörtlichen Funktionszuweisung" (Terfrüchte/Flex 2018: 2970). Die klassische zentralörtliche Funktion besteht in der Bereitstellung von Versorgungseinrichtungen, nicht nur für die ortsansässige Bevölkerung, sondern auch für die Menschen im umliegenden Versorgungsraum. Zentrale Orte weisen daher eine „Bündelung sozialer, kultureller und wirtschaftlicher Einrichtungen auf" (BBSR 2017: 34) und sollen weiterhin bevorzugt Standort von Infrastrukturentwicklung werden (vgl. § 2 Abs. 2 Nr. 3 ROG).

Aus der Perspektive des Schutzes kritischer Infrastrukturen betrachtet, wird mithilfe des Zentrale-OrteKonzepts planmäßig eine räumliche Konzentration potenzieller Schutzgüter/Gefahrenquellen herbeigeführt. Unter der Annahme, dass das Phänomen "geographische Interdependenz" (vgl. Kapitel 3) nicht auf Trassenverläufe beschränkt ist, könnte sich das so erzeugte Muster aus der Perspektive des Schutzes kritischer Infrastrukturen als eher ungünstig darstellen. Darüber hinaus ist zu vermuten, dass besonders viele der überörtlich relevanten Infrastrukturen, die bereits in Zentralen Orten gebündelt sind und weiter dort gebündelt werden sollen, gleichzeitig als kritische Infrastrukturen bewertet werden könnten: Nebenbei, aber gleichsam systematisch, könnten über Zentrale-Orte-Konzepte nicht bloß Infrastrukturen, sondern selektiv gerade kritische Infrastrukturen in Zentralen Orten angesiedelt werden, sodass die Zentralität eines Zentralen Orts auch zu einem gewissen Grad mit der Kritikalität (vgl. Kapitel 2) der dort versammelten Infrastrukturelemente einhergehen würde. Sollte das Zentrale-Orte-Konzept überproportional viele kritische Infrastrukturen adressieren, könnte die Entstehung hoher „,äumlicher Kritikalität“ (vgl. Kapitel 3) zusätzlich begünstigt werden.

Aus den hier aufgestellten Thesen lässt sich eine Reihe von Forschungsfragen ableiten. Die erste Frage setzt an der Rolle räumlicher Nähe im Risikomanagement selbst an: Während die Bündelung linienhafter Infrastrukturtrassen unter Aspekten des Schutzes kritischer Infrastrukturen problematisiert wird (vgl. Kapitel 3), ist dies bislang in Bezug auf die Bündelung punktartiger Infrastrukturelemente nicht der Fall. Eine „signifikante geografische Konzentration von Anlagen" (BBK 2019b: 45) wird bei der Identifizierung kritischer Infrastrukturen allerdings als relevant betrachtet. Darüber hinaus wäre der Zusammenhang zwischen den Bedeutsamkeitsmaßen Zentralität und Kritikalität empirisch zu untersuchen: Erzeugt das Instrument der Zentrale-Orte-Konzepte tat- 
sächlich eine gegebenenfalls sektoral unterschiedlich ausgeprägte räumliche Struktur spezifisch kritischer Infrastrukturen? Wird dadurch eine aus der Sicht des Schutzes kritischer Infrastrukturen problematische "geographische Interdependenz" hervorgebracht oder verstärkt? Daran schließt eine ganze Reihe differenzierterer Forschungsfragen an: In welchem Maße sind kritische Infrastrukturen als zentrengebundene bzw. zentrenprägende Einrichtungen $z u$ betrachten, unterliegen den damit verbundenen, unterschiedlich hohen Konzentrationserfordernissen bzw. zeigen die entsprechenden Effekte (vgl. Flex/Greiving/Terfrüchte 2016: 119-121)? Unterscheiden sich die länderspezifischen Varianten des Zentrale-Orte-Konzepts hinsichtlich ihrer ,Begleiterscheinungen' für den Schutz kritischer Infrastrukturen? Und kann dem Schutz kritischer Infrastrukturen bei deren Ausgestaltung Rechnung getragen werden (z. B. über Ausstattungskataloge, Festlegung von Hierarchiestufen, Bindungswirkung oder Zulässigkeit von Funktionsteilung)?

\subsection{Zentralisierung der Leistungsbereitstellung und Kritikalität von Infrastrukturelementen}

Der Perspektivwechsel funktioniert auch in umgekehrter Richtung, um nach den oft versteckten räumlichen Aspekten in den für den Schutz kritischer Infrastrukturen zentralen Konzepten zu suchen. Beispielsweise spielt bei der Identifizierung von kritischen Infrastrukturelementen nach BBK (2019b) deren Kapazität eine Rolle (vgl. Kapitel 2). Auf welche Leistung ein Infrastrukturelement ausgelegt sein muss, hängt unter anderem damit zusammen, wie viele Elemente an der Bereitstellung einer bestimmten Gesamtleistung beteiligt sind. Wenn mehrere Elemente mit einer jeweils entsprechend geringen Kapazität einen Anteil bereitstellen, wäre mit dem Ausfall eines Elements ein anteilsmäßiger Teilausfall der Gesamtleistung verbunden (der gegebenenfalls sogar durch eine vorübergehende Steigerung der Auslastung verbleibender Elemente kompensiert werden könnte, vgl. Kapitel 6 zu Redundanz). Stellt hingegen im Extremfall nur ein einziges Element mit einer entsprechend hohen Kapazität die Gesamtleistung zur Verfügung, hätte dessen Ausfall auch den Totalausfall der Gesamtleistung zur Folge - die Kritikalität dieses Infrastrukturelements wäre entsprechend hoch einzustufen.

Dieser Zusammenhang bildet den Hintergrund zu dem im Raumordnungsbericht 2011 am Beispiel „zentrale versus dezentrale Wasserversorgung" (BBSR 2012:
54) aufgeworfenen Problem: Wenn die Gesamtmenge benötigten Trinkwassers in einem Versorgungsgebiet dezentral an mehreren Standorten aufbereitet wird, reißt der Ausfall eines Wasserwerks eine Lücke. Diese fällt umso größer aus, je höher dessen Anteil an der Aufbereitung der Gesamtmenge im Versorgungsgebiet ist. Bei einem Höchstmaß an Zentralisierung, wenn ein einziges Wasserwerk mit entsprechend hoher Kapazität die Gesamtmenge bereitstellt, könnte dessen Ausfall den vollständigen Ausfall der Trinkwasserversorgung im Versorgungsgebiet zur Folge haben. Übertragen auf den Bezugsrahmen „Versorgungsgebiet“ schlägt sich der Zusammenhang zwischen Anzahl, Kapazität und Kritikalität von Elementen in einem System in Fragen nach einer eher zentral oder dezentral organisierten Bereitstellung von Daseinsvorsorgeleistungen nieder.

Die planerische Relevanz der Wechselwirkung zwischen Zentralisierung der Leistungserbringung und Kritikalität der daran beteiligten Infrastrukturelemente kommt zum Tragen, wenn in einem Versorgungsgebiet Anzahl und Auslegung von Infrastrukturelementen infrage steht. Dies ist klassischerweise der Fall, wenn beim Leistungsausbau der Daseinsvorsorge entschieden werden muss, ob bestehende Einrichtungen ausgebaut oder durch weitere ergänzt werden. Die Situation entsteht aber auch unter umgekehrten Vorzeichen, wenn mittels „Rücknahmeplanung“ eine „ungeordnete Angebotsrücknahme" (BMVI/BBSR 2016: 14-15) von Daseinsvorsorgeleistungen in der Fläche verhindert werden soll. ${ }^{24}$ Wird in diesem Fall die Anzahl der Infrastrukturelemente reduziert, indem mehrere, für sich genommen ,unkritische Einrichtungen zusammengelegt werden, können ,neue kritische Infrastrukturen entstehen. Umgekehrt kann durch den Erhalt mehrerer Einrichtungen mit jeweils verringerter Kapazität der entgegengesetzte Fall eintreten und vormals als kritisch geltende Infrastrukturelemente diesen Status verlieren. Ob Veränderungen an der Bereitstellung von Daseinsvorsorgeleistungen eher zu einer Zentralisierung oder Dezentralisierung führen, hat also Auswirkungen auf die Kritikalität der beteiligten Infrastrukturelemente und, sofern entsprechende Verfahren etabliert sind (vgl. Kapitel 2), gegebenenfalls auch auf die Anzahl der Elemente, die als kritisch bewertet werden.

24 Die Notwendigkeit, Daseinsvorsorgeplanung auch als Rücknahmeplanung zu begreifen, spiegelt sich raumordnungspolitisch im Leitbild "Daseinsvorsorge Sichern“ (MKRO 2016: 10-13), mit dem sich der Raumordnungsbericht 2017 (BBSR 2017) ausführlich auseinandersetzt. 
Rein unter Kritikalitätsgesichtspunkten ist die Verteilung der Leistungserbringung auf mehr Infrastrukturelemente gegenüber einer Konzentration auf wenige Einrichtungen mit einer entsprechend hohen Kapazität demnach vorteilhaft. Ob und wie dieser hier nur isoliert dargestellte Aspekt in die Gesamtbetrachtung und, im konkreten Fall, auch in die Entscheidungsfindung einfließen könnte, ist damit freilich nicht geklärt. Das Unterhalten mehrerer Einrichtungen kann vergleichsweise höhere Kosten verursachen - das Einhalten von Sicherheitsauflagen für zwar insgesamt weniger, aber dafür als kritisch eingestufte Elemente jedoch auch. Wie können also in wirtschaftlicher Hinsicht Kritikalitätsund Tragfähigkeitsaspekte gegeneinander abgewogen werden? Mehrere in einem Versorgungsraum verteilte Einrichtungen wären nicht nur unter Kritikalitätsgesichtspunkten günstig, sondern würden auch der Erreichbarkeit der betreffenden Daseinsvorsorgeleistung zugutekommen. Befinden sich alle Infrastrukturelemente in nächster Nähe zueinander, stellt sich dieser Effekt nicht ein und der Nachteil "geographischer Interdependenz" könnte den Vorteil mehrerer Elemente aufzehren. Unter welchen Bedingungen können sich also Kritikalitäts- und Erreichbarkeitserwägungen synergetisch zueinander verhalten?

\section{Fazit}

Wie in Kapitel 5 erörtert, dürfte ein Großteil jener „wichtigen Infrastrukturleistungen“, deren "gravierende Störungen und Ausfälle" (BMI 2009: 10) der Schutz kritischer Infrastrukturen adressiert, als Leistungen der Daseinsvorsorge bereitgestellt werden. Dafür spricht zunächst die Gegenüberstellung von Sektoren kritischer Infrastrukturen und Daseinsvorsorgebereichen - auch wenn sich die Überschneidungen nicht eindeutig bemessen lassen (vgl. BBSR 2012: 53). Zudem leitet BBK (2019b) explizit dazu an, in Bereichen der Daseinsvorsorge auch ohne Pendant in den Sektoren nach kritischen Dienstleistungen zu suchen, und einige im Kontext der SARS-CoV2-Pandemie erlassenen Regelungen weisen darauf hin, dass die Sektoreneinteilung zumindest unterhalb des Bundes keine ausschließende Wirkung hat. Demnach ist davon auszugehen, dass viele als kritisch eingestufte Infrastrukturen gleichzeitig als Infrastrukturen der Daseinsvorsorge ihren Dienst versehen und als ,kritische Infrastrukturen der Daseinsvorsorge' im doppelten Sinne als versorgungsrelevant betrachtet werden können.

Es ist naheliegend, dass diese Infrastrukturen in ihrer Funktion als Infrastrukturen der Daseinsvorsorge bereits zum Gegenstand räumlicher Planung wurden und es weiterhin werden. Daher stellt sich die Frage, wie über die räumliche Organisation der Bereitstellung von Versorgungsleistungen die Rahmenbedingungen für den Umgang mit dem Risiko ihres Ausfalls gestaltet werden. Um den Blick für mögliche ,Nebenwirkungen' räumlicher Daseinsvorsorgeplanung für den Schutz kritischer Infrastrukturen zu schärfen, bietet sich ein Perspektivwechsel an, das heißt die Betrachtung von Zielen, Instrumenten und Ansätzen der räumlichen Planung aus dem Blickwinkel des Schutzes kritischer Infrastrukturen (vgl. Kapitel 6). Der ambivalenten Rolle der kritischen Infrastruktur als Schutzgut und Gefahrenquelle wegen taucht ein solcher Perspektivwechsel die Vorgehensweisen der Daseinsvorsorgeplanung zuweilen in ungewohntes Licht. So macht sich die aus raumplanerischer Sicht erstrebenswerte Bündelung überörtlich bedeutender Infrastrukturen in Zentralen Orten der systematischen Verstärkung „räumlicher Kritikalität“ verdächtig (vgl. Kapitel 6.1) und mit der Entscheidung über eine zentrale oder dezentrale Organisation der Bereitstellung von Daseinsvorsorgeleistungen könnte nebenbei die Anzahl kritischer Infrastrukturen beeinflusst werden (vgl. Kapitel 6.2). Die vielfältigen Wechselbeziehungen zwischen räumlicher Daseinsvorsorgeplanung und dem Schutz kritischer Infrastrukturen dürften hinreichend Stoff für einen neuen Diskussionsstrang innerhalb der in Kapitel 3 zusammengefassten fachöffentlichen Auseinandersetzung bieten. Empirisch ausgerichtete Forschungsvorhaben könnten dabei auch der Frage nachgehen, ob die beiden Perspektiven tatsächlich in Planungsprozessen aufeinandertreffen - und welche Argumente dabei von welchen Akteuren mit welchem Erfolg vorgebracht werden.

Danksagung: Die Idee zu diesem Beitrag entstand bei einem Gastaufenthalt am Center for Security Studies (CSS) der Eidgenössischen Technischen Hochschule Zürich. Mein Dank gilt insbesondere Florian Roth und Tim Prior sowie den Mitarbeiterinnen und Mitarbeitern des CCS und dem Deutschen Akademischen Austauschdienst (DAAD). Darüber hinaus danke ich den Gutachterinnen und Gutachtern für ihr wertvolles Feedback zum Manuskript.

\section{Literatur}

ARL - Akademie für Raumforschung und Landesplanung (2016): Daseinsvorsorge und gleichwertige Lebensverhältnisse neu denken. Perspektiven und Handlungsfelder. Hannover. = Positionspapier aus der ARL 108. 
Badura, P. (1966): Die Daseinsvorsorge als Verwaltungszweck der Leistungsverwaltung und der soziale Rechtsstaat. In: Die Öffentliche Verwaltung 19, 17/18, 624-633.

BBK - Bundesamt für Bevölkerungsschutz und Katastrophenhilfe (2012): Schutzkonzepte Kritischer Infrastrukturen im Bevölkerungsschutz. Ziele, Zielgruppen, Bestandteile und Umsetzung im BBK. Bonn. = Wissenschaftsforum 11.

BBK - Bundesamt für Bevölkerungsschutz und Katastrophenhilfe (2019a): BBK-Glossar. Ausgewählte zentrale Begriffe des Bevölkerungsschutzes. Bonn. $=$ Praxis im Bevölkerungsschutz 8.

BBK - Bundesamt für Bevölkerungsschutz und Katastrophenhilfe (2019b): Schutz Kritischer Infrastrukturen. Identifizierung in sieben Schritten. Arbeitshilfe für die Anwendung im Bevölkerungsschutz. Bonn. = Praxis im Bevölkerungsschutz 20.

BBK - Bundesamt für Bevölkerungsschutz und Katastrophenhilfe (2020): 10 Jahre „KRITIS-Strategie“. Einblicke in die Umsetzung der Nationalen Strategie zum Schutz Kritischer Infrastrukturen. Bonn. = Praxis im Bevölkerungsschutz 21.

BBSR - Bundesinstitut für Bau-, Stadt- und Raumforschung (2012): Raumordnungsbericht 2011. Bonn.

BBSR - Bundesinstitut für Bau-, Stadt- und Raumforschung (2017): Raumordnungsbericht 2017. Daseinsvorsorge sichern. Bonn.

Beirat für Raumentwicklung beim Bundesministerium für Verkehr und digitale Infrastruktur (2015): Raumentwicklung und digitale Infrastruktur. Empfehlung, 18. Legislaturperiode. Berlin.

Blotevogel, H. H. (1996): Zentrale Orte: zur Karriere und Krise eines Konzepts in Geographie und Raumplanung. In: Erdkunde 50, 1, 9-25.

BMI - Bundesministerium des Innern (2005): Schutz Kritischer Infrastrukturen - Basisschutzkonzept. Empfehlungen für Unternehmen. Berlin.

BMI - Bundesministerium des Innern (2009): Nationale Strategie zum Schutz Kritischer Infrastrukturen (KRITIS-Strategie). Berlin.

BMI - Bundesministerium des Innern (2011): Schutz Kritischer Infrastrukturen - Risiko- und Krisenmanagement. Leitfaden für Unternehmen und Behörden. Berlin.

BMVBS - Bundesministerium für Verkehr, Bau und Stadtentwicklung (Hrsg.) (2010): Regionale Daseinsvorsorgeplanung. Ein Leitfaden zur Anpassung der öffentlichen Daseinsvorsorge an den demographischen Wandel. Berlin. = Werkstatt: Praxis 64 .

BMVBS - Bundesministerium für Verkehr, Bau und Stadtentwicklung; BBSR - Bundesinstitut für Bau-, Stadt- und Raumforschung (Hrsg.) (2009): Regionalplanerische Ansätze zur Gewährleistung der öffentlichen Daseinsvorsorge. Bonn. = BBSR-Online-Publikation 32/2009.

BMVI - Bundesministerium für Verkehr und digitale Infrastruktur (2017a): Handbuch zur Ausgestaltung der Hochwasservorsorge in der Raumordnung. Berlin. = MORO Praxis 10.

BMVI - Bundesministerium für Verkehr und digitale Infrastruktur (Hrsg.) (2017b): Daseinsvorsorge in der Regionalplanung und Möglichkeiten ihrer formellen und informellen Steuerung. Berlin. = BMVI-Online-Publikation 3/2017.

$\mathrm{BMVI}$ - Bundesministerium für Verkehr und digitale Infrastruktur; BBSR - Bundesinstitut für Bau-, Stadt- und Raumforschung (Hrsg.) (2016): Regionalstrategie Daseinsvorsorge. Leitfaden für die Praxis. Berlin.
Cumbers, A.; Becker, S. (2018): Making sense of remunicipalisation: theoretical reflections on and political possibilities from Germany's Rekommunalisierung process. In: Cambridge Journal of Regions, Economy and Society 11, 3, 503-517. doi: 10.1093/cjres/rsy025

Deutscher Bundestag (1998): „Schlanker Staat": Nächste Schritte. Unterrichtung durch die Bundesregierung. Bundestagsdrucksache 13/10145 vom 19. März 1998. Berlin.

Deutscher Bundestag (2008): Entwurf eines Gesetzes zur Neufassung des Raumordnungsgesetzes und zur Änderung anderer Vorschriften (GeROG). Drucksache 16/10292 vom 22. September 2008. Berlin.

Einig, K. (2008): Regulierung der Daseinsvorsorge als Aufgabe der Raumordnung im Gewährleistungsstaat. In: Informationen zur Raumentwicklung 1/2, 17-40.

Engels, J. I. (2018): Relevante Beziehungen. Vom Nutzen des Kritikalitätskonzepts für Geisteswissenschaftler. In: Engels, J. I.; Nordmann, A. (Hrsg.): Was heißt Kritikalität? Zu einem Schlüsselbegriff der Debatte um Kritische Infrastrukturen. Bielefeld, 17-46. doi: 10.14361/9783839442074-002

Engels, J. I.; Nordmann, A. (2018): Vorwort. In: Engels, J. I.; Nordmann, A. (Hrsg.): Was heißt Kritikalität? Zu einem Schlüsselbegriff der Debatte um Kritische Infrastrukturen. Bielefeld, 7-15. doi: 10.14361/9783839442074-001

Fekete, A. (2011): Common Criteria for the Assessment of Critical Infrastructures. In: International Journal of Disaster Risk Science 2, 1, 15-24. doi: 10.1007/s13753-011-0002-y

Flex, F.; Greiving, S.; Terfrüchte, T. (2016): Strukturen eines (fortentwickelten) Zentrale-Orte-Konzepts. In: Greiving, S.; Flex, F. (Hrsg.): Neuaufstellung des Zentrale-Orte-Konzepts in Nordrhein-Westfalen. Hannover, 106-122. = Arbeitsberichte der ARL 17.

Folkers, A. (2012): Kritische Infrastruktur. In: Marquardt, N.; Schreiber, V. (Hrsg.): Ortsregister. Ein Glossar zu Räumen der Gegenwart. Bielefeld, 154-159. doi: 10.14361/ transcript.9783839419687.154

Folkers, A. (2017): Existential provisions: The technopolitics of public infrastructure. In: Environment and Planning D 35, 5, 855-874. doi: 10.1177/0263775817698699

Folkers, A. (2018a): Das Sicherheitsdispositiv der Resilienz. Katastrophische Risiken und die Biopolitik vitaler Systeme. Frankfurt.

Folkers, A, (2018b): Was ist kritisch an Kritischer Infrastruktur? Kriegswichtigkeit, Lebenswichtigkeit, Systemwichtigkeit und die Infrastrukturen der Kritik. In: Engels, J. I.; Nordmann, A. (Hrsg.): Was heißt Kritikalität? Zu einem Schlüsselbegriff der Debatte um Kritische Infrastrukturen. Bielefeld, 123-154. doi: 10.14361/9783839442074-005

Forsthoff, E. (1938): Die Verwaltung als Leistungsträger. Stuttgart. = Königsberger rechtswissenschaftliche Forschungen 2 .

Forsthoff, E. (1959): Rechtsfragen der leistenden Verwaltung. Stuttgart. = res publica 1 .

Greiving, S. (2011): Methodik zur Festlegung raum- und raumplanungsrelevanter Risiken. In: Pohl, J.; Zehetmair, S. (Hrsg.): Risikomanagement als Handlungsfeld in der Raumplanung. Hannover, 22-30. = Arbeitsmaterial der ARL 357.

Greiving, S. (2018): Strategien der regionalen raumplanerischen Risikovorsorge. In: Kanonier, A.; Rudolf-Miklau, F. (Hrsg.): 
Regionale Risiko Governance: Recht, Politik und Praxis. Wien, 411-425.

Greiving, S.; Flex, F.; Terfrüchte, T. (2015): Vergleichende Untersuchung der Zentrale-Orte-Konzepte in den Ländern und Empfehlungen zu ihrer Weiterentwicklung. In: Raumforschung und Raumordnung 73, 4, 285-297. doi: 10.1007/s13147-0150357-4

Greiving, S.; Hartz, A.; Hurth, F.; Saad, S. (2016): Raumordnerische Risikovorsorge am Beispiel der Planungsregion Köln. In: Raumforschung und Raumordnung 74, 2, 83-99. doi: $10.1007 /$ s13147-016-0387-6

Hartz, A.; Saad, S.; Greiving, S.; Fleischhauer, M.; Hurth, F.; Lindner, C. (2015): Vorsorgendes Risikomanagement in der Regionalplanung. Endbericht (AZ 10.05.06-13.6). Berlin.

Hempel, L.; Kraff, B. D.; Pelzer, R. (2018): Dynamic interdependencies: Problematising criticality assessment in the light of cascading effects. In: International Journal of Disaster Risk Reduction 30, 257-268. doi: 10.1016/j.ijdrr.2018.04.011

inter3 - Institut für Ressourcenmanagement (2019): Analyse von Interdependenzen zwischen KRITIS. Empfehlungen für Praxisakteure aus Versorgungsunternehmen und kommunalen Behörden. Berlin.

Kaufmann, S. (2011): Zivile Sicherheit: Vom Aufstieg eines Topos. In: Hempel, L.; Krasmann, S.; Bröckling, U. (Hrsg.): Sichtbarkeitsregime. Überwachung, Sicherheit und Privatheit im 21. Jahrhundert. Wiesbaden, 101-123. = Leviathan Sonderheft 25.

Kersten, J. (2005): Die Entwicklung des Konzepts der Daseinsvorsorge im Werk von Ernst Forsthoff. In: Der Staat 44, 4, 543-569.

Klingholz, R. (2015): Vielfalt statt Gleichwertigkeit - Die Regionalpolitik braucht eine neue Zielsetzung. In: Informationen zur Raumentwicklung 1, 23-27.

Kloepfer, M. (2015): Handbuch des Katastrophenrechts. Bevölkerungsschutzrecht, Brandschutzrecht, Katastrophenschutzrecht, Katastrophenvermeidungsrecht, Rettungsdienstrecht, Zivilschutzrecht. Baden-Baden. = Schriften zum Katastrophenrecht 9.

Knauff, M. (2004): Der Gewährleistungsstaat: Reform der Daseinsvorsorge. Eine rechtswissenschaftliche Untersuchung unter besonderer Berücksichtigung des ÖPNV. Berlin. = Schriften zum Öffentlichen Recht 976.

KOM - Kommission der Europäischen Gemeinschaften (1996): Leistungen der Daseinsvorsorge in Europa. Mitteilung der Kommission vom 11. September 1996. KOM(96) 443. Brüssel.

Krajewski, M. (2011): Grundstrukturen des Rechts öffentlicher Dienstleistungen. Berlin.

Krings, S. (2018): "Dear Neighbours..." A Comparative Exploration of Approaches to Managing Risks Related to Hazardous Incidents and Critical Infrastructure Outages. In: Erdkunde 72, 2, 103-123. doi: 10.3112/erdkunde.2018.02.03

Lange, C.; Henke, A.; Kariger, M. (2015): Hannover: Einsatzplanung „Kritische Infrastrukturen“. Einsatzplan „KRITIS“ mit Schwerpunkt Stromausfall wurde umgesetzt. In: Brandschutz - Deutsche Feuerwehr-Zeitung 69, 1, 6-10.

Lauwe, P.; Riegel, C. (2008): Schutz Kritischer Infrastrukturen Konzepte zur Versorgungssicherheit. In: Informationen zur Raumentwicklung 1/2, 113-125.

Lenz, S. (2009): Vulnerabilität Kritischer Infrastrukturen. Bonn. = Forschung im Bevölkerungsschutz 4.
Libbe, J. (2012): Rekommunalisierung - empirische Belege und Einordnung in den ökonomischen und rechtlichen Bezugsrahmen. In: Verwaltung und Management 18, 1, 21-33. doi: 10.5771/0947-9856-2012-1-21

Lukitsch, K.; Müller, M.; Stahlhut, C. (2018): Criticality. In: Engels, I. (Hrsg.): Key Concepts for Critical Infrastructure Research. Wiesbaden, 11-20. doi: 10.1007/978-3-658-22920-7_2

Milstein, A. (2018): Daseinsvorsorge. In: ARL - Akademie für Raumforschung und Landesplanung (Hrsg.): Handwörterbuch der Stadt- und Raumentwicklung. Hannover, 361-373.

MKRO - Ministerkonferenz für Raumordnung (2016): Leitbilder und Handlungsstrategien für die Raumentwicklung in Deutschland. Beschlossen von der 41. MKRO am 9. März 2016. Berlin.

Neisser, F. (2016): Kritische Infrastruktur und mobiles Risiko. Eine sozialgeographische Untersuchung auf Basis der AkteurNetzwerk-Theorie. Dissertation an der Rheinischen FriedrichWilhelms-Universität Bonn.

Pleiner, T. (2016): Überplanung von Infrastruktur am Beispiel energiewirtschaftlicher Streckenplanungen unter besonderer Berücksichtigung der Leitungsbündelung. Tübingen. = Schriften zum Infrastrukturrecht 6 .

Pleiner, T. (2018): Die Bündelung von Leitungen beim Energienetzausbau - juristische Sicht. In: Bundesnetzagentur (Hrsg.): Tagungsband Wissenschaftsdialog 2017. Bonn, 44-55.

Pütz, T.; Spangenberg, M. (2006): Zukünftige Sicherung der Daseinsvorsorge. Wie viele Zentrale Orte sind erforderlich? In: Informationen zur Raumentwicklung 6/7, 337-344.

Riegel, C. (2014): Infrastructure resilience through regional spatial planning - prospects of a new legal principle in Germany. In: International Journal of Critical Infrastructures 10, 1, 17-29. doi: 10.1504/IJCIS.2014.059541

Riegel, C. (2015a): Die Berücksichtigung des Schutzes kritischer Infrastrukturen in der Raumplanung. Zum Stellenwert des KRITIS-Grundsatzes im Raumordnungsgesetz. Aachen. = Berichte des Instituts für Stadtbauwesen und Stadtverkehr 59.

Riegel, C. (2015b): Spatial criticality - identifying CIP hot-spots for German regional planning. In: International Journal of Critical Infrastructures 11, 3, 265-277. doi: 10.1504/IJCIS.2015.072157

Rinaldi, S. M.; Peerenboom, J. P.; Kelly, T. K. (2001): Identifying, understanding, and analyzing critical infrastructure interdependencies. In: IEEE Control Systems Magazine 21, 6 , 11-25. doi: 10.1109/37.969131

Runkel, P. (2018): Ziele, Grundsätze und sonstige Erfordernisse der Raumordnung. In: ARL - Akademie für Raumforschung und Landesplanung (Hrsg.): Handwörterbuch der Stadt- und Raumentwicklung. Hannover, 2989-2999.

Scheidemann, D. (1991): Der Begriff Daseinsvorsorge. Ursprung, Funktion und Wandlungen der Konzeption Ernst Forsthoffs. Göttingen. = Göttinger politikwissenschaftliche Forschungen 5.

Schmidt, M. (2013): Regional Governance und Infrastruktur Kooperationen in der Wasserver- und Abwasserentsorgung am Beispiel der Stadtregionen Frankfurt/M, Berlin und Ruhr. Detmold.

Schmitt, H. C. (2019): Systemisches Kaskadenpotenzial von KRITIS-Teilsektoren. In: Informationen zur Raumentwicklung 4, 48-61.

Scholles, F. (2018): Bündelung aus planerischer Sicht. In: Bundesnetzagentur (Hrsg.): Tagungsband Wissenschaftsdia$\log 2017$. Bonn, 36-42. 
Schulze, T. (2006): Bedingt abwehrbereit. Schutz kritischer Informationsinfrastrukturen in Deutschland und den USA. Wiesbaden. doi: 10.1007/978-3-531-90130-5

Sonntag, M. (2005): IT-Sicherheit kritischer Infrastrukturen. Von der Staatsaufgabe zur rechtlichen Ausgestaltung. München. = Schriftenreihe Information und Recht 48.

Steinführer, A. (2015): Bürger in der Verantwortung. Veränderte Akteursrollen in der Bereitstellung ländlicher Daseinsvorsorge. In: Raumforschung und Raumordnung 73, 1, 5-16. doi: 10.1007/ s13147-014-0318-3

Terfrüchte, T. (2019): Gleichwertige Lebensverhältnisse zwischen Raumordnung und Regionalpolitik. In: Wirtschaftsdienst 99, 13, 24-30. doi: 10.1007/s10273-019-2428-6

Terfrüchte, T.; Flex, F. (2018): Zentraler Ort. In: ARL - Akademie für Raumforschung und Landesplanung (Hrsg.): Handwörterbuch der Stadt- und Raumentwicklung. Hannover, 2969-2979.

van Laak, D. (1999): Der Begriff "Infrastruktur“ und was er vor seiner Erfindung besagte. In: Archiv für Begriffsgeschichte 41, 280-299.

van Laak, D. (2001): Infra-Strukturgeschichte. In: Geschichte und Gesellschaft 27, 3, 367-393.

WD - Wissenschaftlicher Dienst des Deutschen Bundestags (2012): Internet als Teil der staatlichen Daseinsvorsorge. Ausarbeitung WD 10-3000/115-11. Berlin.

Wiater, P. (2013): Sicherheitspolitik zwischen Staat und Markt. Der Schutz kritischer Infrastrukturen. Baden-Baden. = Sicherheit und Gesellschaft. Freiburger Studien des Center for Security and Society 6.

Wiater, P. (2017): Bürger und Unternehmen als Akteure der Zivilen Sicherheit. In: Gusy, C.; Kugelmann, D.; Würtenberger, T. (Hrsg.): Rechtshandbuch Zivile Sicherheit. Berlin, 225-245. doi: 10.1007/978-3-662-53289-8_12 